\title{
Seven pivotal theorems of Fourier analysis, signal analysis, numerical analysis and number theory: their interconnections
}

\author{
P. L. Butzer • M. M. Dodson • P. J. S. G. Ferreira • \\ J. R. Higgins • G. Schmeisser • R. L. Stens
}

Received: 13 June 2014 / Revised: 8 September 2014 / Accepted: 23 September 2014 /

Published online: 17 October 2014

(C) The Author(s) 2014. This article is published with open access at SpringerLink.com

\begin{abstract}
The present paper deals mainly with seven fundamental theorems of mathematical analysis, numerical analysis, and number theory, namely the generalized Parseval decomposition formula (GPDF), introduced 15 years ago, the well-known approximate sampling theorem (ASF), the new approximate reproducing kernel theorem, the basic Poisson summation formula, already known to Gauß, a newer version of the GPDF having a structure similar to that of the Poisson summation formula, namely,
\end{abstract}

In memory of Wolfgang Splettstößer (1950-2013), a pioneer in sampling analysis and valued colleague.

Communicated by S. K. Jain.

P. L. Butzer · R. L. Stens ( ()

Lehrstuhl A für Mathematik, RWTH Aachen University, 52056 Aachen, Germany

e-mail: stens@matha.rwth-aachen.de

P. L. Butzer

e-mail: butzer@rwth-aachen.de

M. M. Dodson

Department of Mathematics, University of York, York YO1O 5DD, UK

e-mail: mmd1@york.ac.uk

P. J. S. G. Ferreira

IEETA/DETI, Universidade de Aveiro, 3810-193 Aveiro, Portugal

e-mail: pjf@ua.pt

J. R. Higgins

I.H.P., 4 rue du Bary, 11250 Montclar, France

e-mail: rhiggins11@gmail.com

G. Schmeisser

Department of Mathematics, University of Erlangen-Nuremberg, 91058 Erlangen, Germany

e-mail: schmeisser@mi.uni-erlangen 
the Parseval decomposition-Poisson summation formula, the functional equation of Riemann's zeta function, as well as the Euler-Maclaurin summation formula. It will in fact be shown that these seven theorems are all equivalent to one another, in the sense that each is a corollary of the others. Since these theorems can all be deduced from each other, one of them has to be proven independently in order to verify all. It is convenient to choose the ASF, introduced in 1963. The epilogue treats possible extensions to the more general contexts of reproducing kernel theory and of abstract harmonic analysis, using locally compact abelian groups. This paper is expository in the sense that it treats a number of mathematical theorems, their interconnections, their equivalence to one another. On the other hand, the proofs of the many intricate interconnections among these theorems are new in their essential steps and conclusions.

Keywords Bandlimited and non-bandlimited functions - Sampling theorem . Parseval formula $\cdot$ Reproducing kernel formula · Poisson's summation formula · Riemann's zeta function · Euler-Maclaurin summation formula

Mathematics Subject Classfication $\quad 30 \mathrm{D} 10 \cdot 94 \mathrm{~A} 20 \cdot 41 \mathrm{~A} 80 \cdot 42 \mathrm{~A} 38 \cdot 30 \mathrm{D} 05$

\section{Contents}

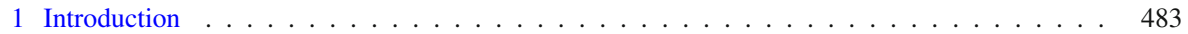

1.1 Generalized Parseval decomposition formula (GPDF) . . . . . . . . . . . . . 483

1.2 Approximate sampling formula (ASF) . . . . . . . . . . . . . . . . . . 484

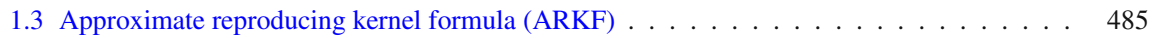

1.4 Poisson's summation formula $(\mathrm{PSF}) \ldots \ldots \ldots \ldots$. . . . . . . . . . . . 486

1.5 Parseval decomposition-Poisson summation formula (PDPS) . . . . . . . . . . . . 486

1.6 Functional equation of the Riemann zeta function (FERZ) . . . . . . . . . . . . . . . . . . 487

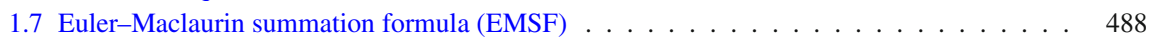

2 Notations and side results . . . . . . . . . . . . . . . . . . . . . . . 490

2.1 Notations . . . . . . . . . . . . . . . . . . . . . . . . . . . . 490

2.2 Results on Fourier analysis . . . . . . . . . . . . . . . . . . . . . . . . . 490

2.3 The sampling series $s_{w} f \ldots \ldots \ldots \ldots \ldots \ldots \ldots \ldots \ldots \ldots$

2.4 Remarks on Poisson's summation formula . . . . . . . . . . . . . . . . . . . . . . . 494

3 Equivalence of the decomposition formula and four summation formulae . . . . . . . . . . 495

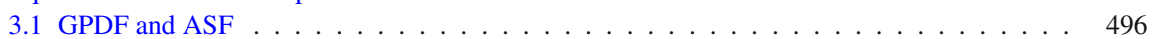

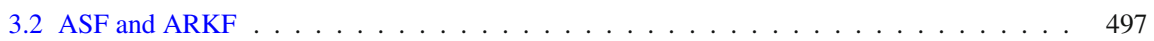

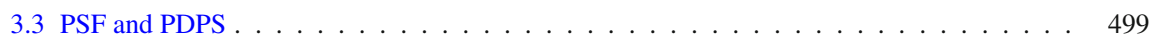

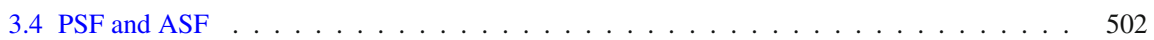

$4 \mathrm{PSF}$ and the functional equation for $\zeta(s) \ldots \ldots \ldots \ldots \ldots \ldots 4 \ldots \ldots \ldots$

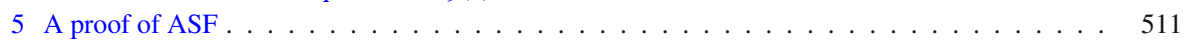

6 Epilogue: some possible generalizations . . . . . . . . . . . . . . . . . . . 513

6.1 Reproducing kernel theory . . . . . . . . . . . . . . . . . . . . . . . . 513

6.2 Abstract harmonic analysis . . . . . . . . . . . . . . . . . . . 515

6.3 Abstract sampling theory . . . . . . . . . . . . . . . . . . . . 517

6.4 Sampling in Hilbert spaces, on Riemannian manifolds and graphs . . . . . . . . . . . . 520

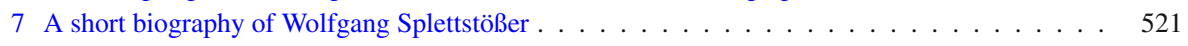

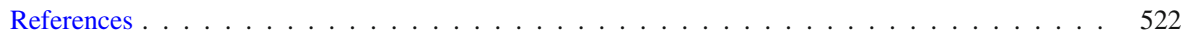




\section{Introduction}

A provoking result of Fourier analysis has turned up in the past 15 years, namely

\subsection{Generalized Parseval decomposition formula (GPDF)}

For $f \in F^{2} \cap S_{w}^{1}, w>0$, and $g \in F^{2}$, there holds $R_{w} f \in L^{2}(\mathbb{R})$ and

$$
\begin{aligned}
& \int_{\mathbb{R}} f(u) \bar{g}(u) d u=\frac{1}{w} \sum_{k \in \mathbb{Z}} f\left(\frac{k}{w}\right) \bar{g}\left(\frac{k}{w}\right) \\
& -\frac{1}{w} \sum_{k \in \mathbb{Z}} f\left(\frac{k}{w}\right) \frac{1}{\sqrt{2 \pi}} \int_{|v| \geq \pi w} \widehat{\bar{g}}(v) \mathrm{e}^{i k v / w} d v+\int_{\mathbb{R}}\left(R_{w} f\right)(u) \bar{g}(u) d u,
\end{aligned}
$$

where

$$
\left(R_{w} f\right)(t):=\frac{1}{\sqrt{2 \pi}} \sum_{k \in \mathbb{Z}}\left(1-\mathrm{e}^{-i 2 \pi k w t}\right) \int_{(2 k-1) \pi w}^{(2 k+1) \pi w} \widehat{f}(v) \mathrm{e}^{i v t} d v \quad(t \in \mathbb{R})
$$

Observe that $\lim _{w \rightarrow \infty}\left(R_{w} f\right)(t)=0$ uniformly for $t \in \mathbb{R}$, since

$$
\left|\left(R_{w} f\right)(t)\right| \leq \sqrt{\frac{2}{\pi}} \int_{|v| \geq \pi w}|\widehat{f}(u)| d u
$$

Here $F^{2}$ and $F^{2} \cap S_{w}^{1}$ are suitable subspaces of $L^{2}(\mathbb{R})$, and $\widehat{f}$ denotes the Fourier transform (for exact definitions see Sect. 2.1).

Formula (1.1), first established in [17,18], can be said to be intermediate between the classical General Parseval/Plancherel formula (or power/energy theorem) for $L^{2}(\mathbb{R})$ functions, namely

$$
\int_{\mathbb{R}} f(u) \bar{g}(u) d u=\int_{\mathbb{R}} \widehat{f}(v) \widehat{g}(v) d v \quad\left(f, g \in L^{2}(\mathbb{R})\right),
$$

and the special case of (1.1) for bandlimited $L^{2}(\mathbb{R})$-functions, i.e., for functions $f, g \in$ $\widehat{B}_{\pi w}^{2}$ (see Sect. 2.1), namely,

$$
\int_{\mathbb{R}} f(u) \bar{g}(u) d u=\frac{1}{w} \sum_{k \in \mathbb{Z}} f\left(\frac{k}{w}\right) \bar{g}\left(\frac{k}{w}\right)=\int_{\mathbb{R}} \widehat{f}(v) \bar{g}(v) d v \quad\left(f, g \in \widehat{B}_{\pi w}^{2}\right) .
$$

In fact, formula (1.1) is the extension of (1.4) from $\widehat{B}_{\pi w}^{2}$ to a larger subclass of $L^{2}(\mathbb{R})$. 
Note that if $g \in \widehat{B}_{\pi w}^{2}$, then the hypotheses of GPDF are satisfied and the second series on the right-hand side of (1.1) vanishes, and so (1.1) reduces to

$$
\int_{\mathbb{R}} f(u) \bar{g}(u) d u=\frac{1}{w} \sum_{k \in \mathbb{Z}} f\left(\frac{k}{w}\right) \bar{g}\left(\frac{k}{w}\right)+\int_{\mathbb{R}}\left(R_{w} f\right)(u) \bar{g}(u) d u .
$$

Moreover, if, in addition, $f \in \widehat{B}_{\pi w}^{2}$, then the integral on the right-hand side of (1.5) also vanishes since $\left(R_{w} f\right)(u)=0$, and so (1.5) reduces to the (classical) General Parseval formula (GPF) for $f, g \in \widehat{B}_{\pi w}^{2}, w>0$, already stated in (1.4). In this sense the latter two terms in GPDF (1.1) can be regarded as remainder terms.

In this instance the assumption $f \in S_{w}^{1}$ is implicitly contained in the hypothesis $f \in \widehat{B}_{\pi w}^{2}$; see Sect. 2.1.

The second formula to be considered is the approximate sampling formula (ASF) treated in Weiss [83], Brown [11,12] and Butzer-Splettstößer [22]; see also [84, pp. 64-66].

\subsection{Approximate sampling formula (ASF)}

For $f \in F^{2} \cap S_{w}^{1}, w>0$, we have

$$
f(t)=\sum_{k \in \mathbb{Z}} f\left(\frac{k}{w}\right) \operatorname{sinc}(w t-k)+\left(R_{w} f\right)(t) \quad(t \in \mathbb{R})
$$

the series converging absolutely and uniformly on $\mathbb{R}$, where

$$
\operatorname{sinc} t:= \begin{cases}\frac{\sin \pi t}{\pi t}, & t \in \mathbb{R} \backslash\{0\} \\ 1, & t=0 .\end{cases}
$$

For the important role played by the sinc-function in mathematics see [80,81].

The ASF for not necessarily bandlimited functions generalizes the classical version

$$
f(t)=\sum_{k \in \mathbb{Z}} f\left(\frac{k}{w}\right) \operatorname{sinc}(w t-k) \quad(t \in \mathbb{R})
$$

of the sampling formula for $f \in \widehat{B}_{\pi w}^{2}$.

In signal analysis, convergence the sampling series is usually defined via the limit of the symmetric partial sums, i. e., $\lim _{N \rightarrow \infty} \sum_{|k| \leq N} f(k / w) \operatorname{sinc}(w t-k)$ [see (2.20)], reflecting the mode of convergence of complex Fourier series (see, e. g., [41, pp. 5-6]). In particular, it is the usual approach in sampling theory, where the ASF holds for functions in $F^{2}$. In the present case, however, the focus is on the equivalence of the validity of the ASF with other results, in particular with the PSF (see Sect. 3.4). 
Whereas to proceed from (1.7) to (1.6) one adds the "error" term $\left(R_{w} f\right)(t)$, to go from (1.4) to (1.1) one has to add two "error" terms.

To make the foregoing generalization fully clear, the ASF yields the GPDF and, conversely, the GPDF for just one special function will yield the ASF; thus the two formulae are truly equivalent to another, as already shown in [18].

A third formula belonging to our grouping of six equivalences in Fig. 1 brings us into contact with the theory of Hilbert spaces with reproducing kernel, an important branch of functional analysis and complex function theory having many areas of applications, with a long history. See, e.g., [3,37,46,62].

It is well known that $\widehat{B}_{\pi w}^{2}$ is a sub-Hilbert space of $L^{2}(\mathbb{R})$ and is, furthermore, an example of a Hilbert space with reproducing kernel. It is a special case of Saitoh's presentation (see, e.g., [41, Ch. 3], [77, pp. 9, 194]). The reproducing kernel is $(t, u) \mapsto$ $w$ sinc $w(t-u)$ and the reproducing kernel formula (RKF), expressing the reproduction of $f \in \widehat{B}_{\pi w}^{2}$ from itself, that is, all of its values, is

$$
f(t)=w \int_{\mathbb{R}} f(u) \operatorname{sinc} w(t-u) d u=\int_{\mathbb{R}} f\left(\frac{\kappa}{w}\right) \operatorname{sinc}(w t-\kappa) d \kappa \quad(t \in \mathbb{R}) .
$$

The second integral in (1.8) is just the first integral rearranged to give a precise integral analogue of the series in (1.7), which may be thought of as a "discrete reproducing kernel formula", expressing the reproduction of $f$ from a discrete subset of its values.

In view of the foregoing equivalence $\mathrm{GPDF} \Leftrightarrow \mathrm{ASF}$, the question arises whether there can be established a generalized version of RKF, thus one for non-bandlimited $L^{1}(\mathbb{R})$-functions, which is equivalent to the former two. In fact, one basic aim of this paper is to introduce the new approximate reproducing kernel formula, namely

\subsection{Approximate reproducing kernel formula (ARKF)}

Let $f \in F^{2} \cap S_{w}^{1}$ with $w>0$. Then $R_{w} f \in L^{2}(\mathbb{R})$ and

$$
f(t)=w \int_{\mathbb{R}} f(u) \operatorname{sinc} w(t-u) d u+\left(R_{w} f\right)(t)-w \int_{\mathbb{R}}\left(R_{w} f\right)(u) \operatorname{sinc} w(t-u) d u .
$$

Clearly, if $f \in \widehat{B}_{\pi w}^{2}$, then again $R_{w} f=0$, and so we obtain the (classical) reproducing kernel formula RKF, namely (1.8). Thus (1.9) represents the classical RKF plus two remainder terms.

There is a fourth formula which is fundamental in a variety of mathematical fields, namely the truly well-known Poisson summation formula (PSF). But for purposes of precision let us state the version we are using. 
1.4 Poisson's summation formula (PSF)

Let $f \in L^{1}(\mathbb{R})$ such that $\widehat{f} \in S_{w}^{1}$ for some $w>0$; then

$$
\sqrt{2 \pi} w \sum_{k \in \mathbb{Z}} f(x+2 k \pi w)=\sum_{k \in \mathbb{Z}} \widehat{f}\left(\frac{k}{w}\right) e^{i k x / w} \quad \text { (a.e.). }
$$

Now to the fifth formula which is actually a different version of GPDF but has a structure similar to that of the Poisson summation formula, however not for one function $f$, but for the product of two, namely $f \bar{g}$ on the left-hand side, and the product of their Fourier transforms on the right-hand side.

\subsection{Parseval decomposition-Poisson summation formula (PDPS)}

For $f \in F^{2} \cap S_{w}^{1}, w>0, \sigma>0$, and $g \in F^{2}$, there holds

$$
\begin{aligned}
\frac{1}{w} \sum_{k \in \mathbb{Z}} f\left(\frac{k}{w}\right) \bar{g}\left(\frac{k}{w}\right) e^{i k x / w}= & \sum_{k \in \mathbb{Z}} \int_{-\sigma}^{\sigma} \widehat{f}(v-x+2 k \pi w) \overline{\widehat{g}}(v) d v \\
& +\frac{1}{w} \sum_{k \in \mathbb{Z}} f\left(\frac{k}{w}\right) \frac{1}{\sqrt{2 \pi}} \int_{|v| \geq \sigma} \widehat{\bar{g}}(v) e^{i k(v+x) / w} d v
\end{aligned}
$$

almost everywhere on $\mathbb{R}$. Furthermore, if rather $g \in F^{1}$ instead of $g \in F^{2}$, then the formula holds for all $x \in \mathbb{R}$.

PDPS can in fact be interpreted as an approximate form of PSF for a product $f \bar{g}$ in which $g$ takes the role of an approximately bandlimited function. Indeed, the second series on the right-hand side of PDPS may be seen as an error term depending on the deviation of $g$ from a bandlimited function. If $g$ is bandlimited to $[-\sigma, \sigma]$, i. e. $g \in \widehat{B}_{\sigma}^{2}$, then PDPS reduces to

$$
\frac{1}{w} \sum_{k \in \mathbb{Z}} f\left(\frac{k}{w}\right) \bar{g}\left(\frac{k}{w}\right) e^{i k x / w}=\sum_{k \in \mathbb{Z}} \int_{-\sigma}^{\sigma} \widehat{f}(v-x+2 k \pi w) \widehat{g}(v) d v
$$

holding almost everywhere on $\mathbb{R}$ when $g \in F^{2}$ and everywhere if rather $g \in F^{1}$.

A particular form of PDPS had already been used in [18] in one of the proofs, namely the form

$$
\begin{aligned}
\frac{1}{w} \sum_{k \in \mathbb{Z}} f\left(\frac{k}{w}\right) \bar{g}\left(\frac{k}{w}\right)= & \sum_{k \in \mathbb{Z}} \int_{-\pi w}^{\pi w} \widehat{f}(v+2 k \pi w) \widehat{g}(v) d v \\
& +\frac{1}{w} \sum_{k \in \mathbb{Z}} f\left(\frac{k}{w}\right) \frac{1}{\sqrt{2 \pi}} \int_{|v| \geq \pi w} \widehat{\bar{g}}(v) \mathrm{e}^{i k v / w} d v,
\end{aligned}
$$


provided $f \in F^{2} \cap S_{w}^{1}$ and $g \in F^{1}$. It is the case $x=0, \sigma=\pi w$ of (1.11), noting

$$
\int_{-\sigma}^{\sigma} \widehat{f}(v-x+2 k \pi w) \bar{g}(v) d v=\int_{2 k \pi w-x-\sigma}^{2 k \pi w-x+\sigma} \widehat{f}(v) \bar{g}(v+x-2 k \pi w) d v .
$$

In fact, PDPS in the form (1.13) is just a different representation of GPDF; see [18] for the details.

Of interest is a version of (1.13) with $g$ replaced by $\widehat{g}$, the Fourier transform of a function $g \in L^{1}(\mathbb{R}) \cap L^{2}(\mathbb{R})$, thus $\widehat{g} \in F^{2}$. The result can be regarded as a discretization of the classical Fourier exchange formula (2.5).

Just as for GPDF, the authors have also never met PDPS in the literature; both formulae also do not seem to be particular cases of specific trace formulae known to the authors.

Next to our sixth formula, the famous

\subsection{Functional equation of the Riemann zeta function (FERZ)}

For the Riemann zeta function, defined by

$$
\zeta(s):=\sum_{k=1}^{\infty} \frac{1}{k^{s}} \quad(s=\sigma+i \tau \in \mathbb{C}, \sigma>1)
$$

and for $\sigma \leq 1$ by analytic continuation, there holds for all $s \in \mathbb{C}$ the equation

$$
\pi^{-\frac{s}{2}} \Gamma\left(\frac{s}{2}\right) \zeta(s)=\pi^{-\frac{1-s}{2}} \Gamma\left(\frac{1-s}{2}\right) \zeta(1-s) \quad(s \in \mathbb{C})
$$

or, equivalently,

$$
2(2 \pi)^{-s} \cos \left(\frac{\pi s}{2}\right) \Gamma(s) \zeta(s)=\zeta(1-s) .
$$

In fact, we shall reprove the well-known equivalence FERZ $\Leftrightarrow$ PSF (see the literature cited in Sect. 4) in great detail, in particular in regard to the interchanges of the orders of summation and/or integration occurring. What is remarkable about this equivalence is that in the direction FERZ $\Rightarrow$ PSF, a very particular result, namely the functional equation for $\zeta(s)$, implies a very general result, the PSF for a large class of functions. In other words, a formula of number theory, (externally) unrelated to periodicity and Fourier series, can yield a basic, general formula of analysis, one connecting Fourier series and integrals.

It is well-known that the FERZ is equivalent to the second famous functional equation, namely FEJT

$$
\vartheta\left(-\frac{1}{z}\right)=(-i z)^{\frac{1}{2}} \vartheta(z)
$$


satisfied by Jacobi's $\vartheta$-function

$$
\vartheta(z)=1+2 \sum_{k=1}^{\infty} \exp \left(i \pi k^{2} z\right)=\sum_{k=-\infty}^{\infty} \exp \left(i \pi k^{2} z\right)
$$

The function $\vartheta$, defined on the upper half-plane $H:=\{z \in \mathbb{C}$; $\Im z>0\}$, is analytic in $H$ and periodic with period 2.

As to the proofs of FERZ $\Rightarrow$ FEJT see e.g. Hamburger [36, pp. 136-137], and for the converse Riemann [74]. In the matter see also [44].

It is well known that an application of PSF to the Gauß kernel of (2.12) yields FEJT (see [20, p. 204]).

Furthermore, Dedekind's $\eta$-function (1877) of elliptic modular functions,

$$
\eta(z)=\exp \left(\frac{i \pi z}{12}\right) \prod_{k=1}^{\infty}(1-\exp (i 2 \pi k z))
$$

analytic on $H$, satisfies the same functional equation (1.17), namely FEDE (cf. [2, pp. 47-50]),

$$
\eta\left(-\frac{1}{z}\right)=(-i z)^{\frac{1}{2}} \eta(z)
$$

Finally to our seventh formula, discovered by Euler in connection with the so-called Basel problem, i.e., with determining $\zeta(2)$ in modern terminology.

\subsection{Euler-Maclaurin summation formula (EMSF)}

For $n, r \in \mathbb{N}$ and $f \in C^{(2 r)}[0, n]$, we have

$$
\begin{aligned}
\sum_{k=0}^{n} f(k)= & \int_{0}^{n} f(x) d x+\frac{1}{2}[f(0)+f(n)]+\sum_{k=1}^{r} \frac{B_{2 k}}{(2 k) !}\left[f^{(2 k-1)}(n)-f^{(2 k-1)}(0)\right] \\
& +(-1)^{r} \sum_{k=1}^{\infty} \int_{0}^{n} \frac{e^{i 2 \pi k t}+e^{-i 2 \pi k t}}{(2 \pi k)^{2 r}} f^{(2 r)}(t) d t
\end{aligned}
$$

where $B_{2 k}$ are the Bernoulli numbers.

The implications to be established in this paper are indicated by the arrows in Fig. 1. For a proof of ASF $\Leftrightarrow$ EMSF the reader is referred to [16]. Thus each of the seven formulae GPDF, ASF, ARKF, PSF, PDPS, FERZ and EMSF is equivalent to each other, in the sense that each is a corollary of each of the others.

This means that three different, particular results of number theory (FERZ, FEJT, FEDE) are equivalent to six general summation formulae of Fourier analysis, signal analysis and numerical analysis, namely PSF, PDPS, GPDF, ASF, ARKF, EMSF.

Section 2 is devoted to notations and the side results needed. In Sect. 3 we prove a first grouping of equivalences, namely, GPDF $\Leftrightarrow \mathrm{ASF}$, and then $\mathrm{ASF} \Leftrightarrow \mathrm{ARKF}$, $\mathrm{PSF} \Leftrightarrow \mathrm{PDPS}$, and finally $\mathrm{ASF} \Leftrightarrow \mathrm{PSF}$. The latter three equivalences are new results 
Fig. 1 The implications to be proved

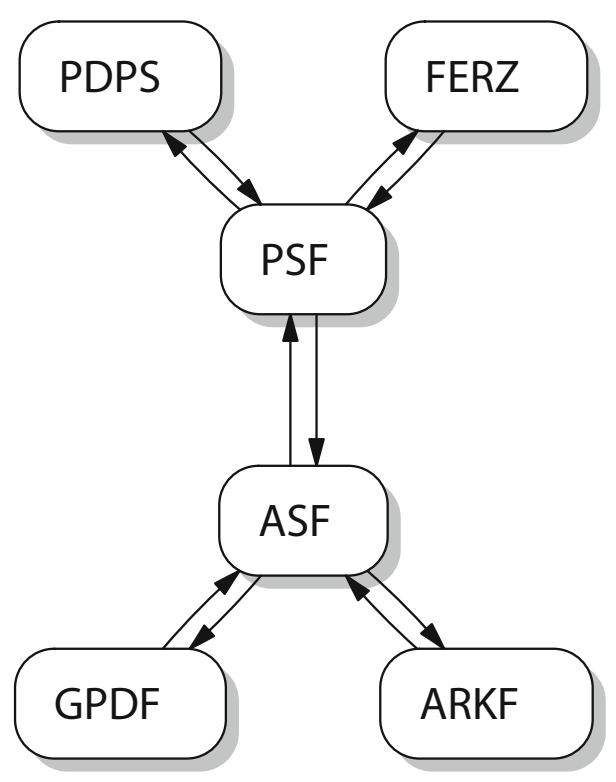

of this paper. Section 4 is concerned with the equivalence of PSF and FERZ, and in Sect. 5 gives a proof of ASF independent of the other formulae of this paper.

The epilogue of Sect. 6, with the topic abstract settings, deals with the possibility of presenting this paper in a setting based on reproducing kernel (r.k.) theory, and also in terms of abstract harmonic analysis, using locally compact abelian groups.

In the present paper, dealing with the grouping (A): GPDF, ASF, ARKF, PSF, PDPS, FERZ, as well as the Euler-Maclaurin summation formula EMSF (handled in [16]) —all for non-bandlimited functions - the basic result is that all seven theorems are equivalent amongst themselves. On the other hand, in our joint paper [15], treating the grouping (B), namely the classical sampling theorem CSF, the general Parseval formula GPF (i. e., the GPDF for bandlimited functions), the reproducing kernel formula GPF (i. e., GPDF for bandlimited functions) and the PSF - all for bandlimited functions - it was shown that the latter four theorems are also all equivalent amongst themselves.

However in our article [14] it was shown that the approximate sampling theorem, the ASF, is equivalent to the classical sampling theorem CSF. Thus the conclusion of the present paper, together with the papers $[15,16]$ and [14], is the full equivalence of the groupings (A) and (B). In other words, the seven theorems for non-bandlimited functions are all equivalent to the corresponding ones for the classical bandlimited functions.

Whereas the majority of the assertions of the theorems in question have been stated and established in earlier papers by a (part) of the present authors, the proofs of the many delicate interconnections between those of grouping (A), the chief topic of the paper, are new in their essential steps and conclusions, as referred to.

Electrical engineers use bandlimited functions as a mathematical model which is a rather severe restriction. In fact there do not exist signals which are simultaneously bandlimited and duration-limited (time-limited). Hence engineers work intuitively with 
approximately bandlimited functions; these are covered by our non-bandlimited theorems. Thus our paper can also be regarded as a further justification of what electrical engineers do in their real life work.

\section{Notations and side results}

\subsection{Notations}

The Fourier transform of $f \in L^{p}(\mathbb{R})$ with $p=1$ or $p=2$ is defined by

$$
\widehat{f}(v):=\frac{1}{\sqrt{2 \pi}} \int_{\mathbb{R}} f(u) \mathrm{e}^{-i v u} d u \quad(v \in \mathbb{R}),
$$

the integral being understood as the limit in the $L^{2}(\mathbb{R})$-norm for $p=2$. We shall consider the following function spaces, namely

$$
F^{p}:=\left\{f \in L^{p}(\mathbb{R}) \cap C(\mathbb{R}) ; \widehat{f} \in L^{1}(\mathbb{R})\right\} \quad(p=1,2),
$$

where $C(\mathbb{R})$ denotes the space of all uniformly continuous and bounded functions on $\mathbb{R}$, and

$$
S_{w}^{p}:=\left\{f: \mathbb{R} \rightarrow \mathbb{C} ;\left(f\left(\frac{k}{w}\right)\right)_{k \in \mathbb{Z}} \in \ell^{p}(\mathbb{Z})\right\} \quad(w>0,1 \leq p \leq \infty) .
$$

There holds $F^{1} \subset F^{2}$ and $S_{w}^{1} \subset S_{w}^{p_{1}} \subset S_{w}^{p_{2}} \subset S_{w}^{\infty}$ for $1 \leq p_{1} \leq p_{2} \leq \infty$.

The Bernstein spaces $\widehat{B}_{\sigma}^{p}$ for $p=1,2$ and $\sigma>0$ are defined in terms of the Fourier transform via

$$
\widehat{B}_{\sigma}^{p}:=\left\{f \in L^{p}(\mathbb{R}) ; \widehat{f}(v)=0 \text { a.e. outside }[-\sigma, \sigma]\right\}
$$

Since the Fourier transform of a function $f \in \widehat{B}_{\sigma}^{p}$ has support on a set of finite measure, those functions are also called "bandlimited". One has the inclusions $\widehat{B}_{\sigma}^{1} \subset \widehat{B}_{\sigma}^{2}$ and $\widehat{B}_{\sigma}^{p} \subset F^{p} \cap S_{w}^{1} \subset F^{p} \cap S_{w}^{p}, p=1,2$; see [64, pp. 123,126].

\subsection{Results on Fourier analysis}

Let us list some well-known results from Fourier analysis; see any textbook in the matter, e.g., [20, Chapt. 5.1 and 5.2].

Proposition 1 (a) If $f \in L^{p}(\mathbb{R}), p=1,2$, then for each $h \in \mathbb{R}$,

$$
\begin{aligned}
& {[f(\cdot+h)] \widehat{(v)}=\widehat{f}(v) e^{i h v}} \\
& {\left[e^{-i h \cdot} f(\cdot) \widehat{]}(v)=\widehat{f}(v+h)\right.}
\end{aligned}
$$

for all $v \in \mathbb{R}$ in case $p=1$ and a.e. in case $p=2$. 
(b) If $f \in L^{1}(\mathbb{R}), g \in L^{p}(\mathbb{R}), p=1,2$, then the convolution

$$
(f * g)(t):=\frac{1}{\sqrt{2 \pi}} \int_{\mathbb{R}} f(u) g(t-u) d u
$$

belongs to $L^{p}(\mathbb{R})$, and for the Fourier transform one has the convolution theorem

$$
(f * g) \widehat{)}(v)=\widehat{f}(v) \widehat{g}(v)
$$

for all $v \in \mathbb{R}$ in case $p=1$ and a.e. in case $p=2$.

(c) If $f, g \in L^{p}(\mathbb{R}), p=1,2$, then there holds the exchange formula

$$
\int_{\mathbb{R}} f(v) \widehat{g}(v) d v=\int_{\mathbb{R}} \widehat{f}(v) g(v) d v
$$

(d) If $f \in L^{2}(\mathbb{R})$, then $\widehat{f}$ also belongs to $L^{2}(\mathbb{R})$ and $\|f\|_{L^{2}(\mathbb{R})}=\|\widehat{f}\|_{L^{2}(\mathbb{R})}$. Furthermore, there holds the inversion formula

$$
f(t)=\int_{\mathbb{R}} \widehat{f}(v) e^{i v t} d v=\widehat{\widehat{f}}(-t) \quad \text { a.e. }
$$

where the integral is again understood as the limit in $L^{2}(\mathbb{R})$-norm. If $f \in F^{p}$ with $p=1$ or $p=2$, then the integral in (2.6) exists as an ordinary Lebesgue integral and both equalities hold for all $t \in \mathbb{R}$.

Lemma 1 There hold the formulae

$$
\begin{array}{ll}
\frac{1}{\sqrt{2 \pi} w} \operatorname{rect}\left(\frac{\cdot}{w}\right) \hat{(}(v)=\operatorname{sinc}(w v) & (v \in \mathbb{R}), \\
\operatorname{sinc}(w \cdot)(v)=\frac{1}{\sqrt{2 \pi} w} \operatorname{rect}\left(\frac{v}{w}\right) & (v \in \mathbb{R})
\end{array}
$$

with the rectangle function

$$
\operatorname{rect}(t):= \begin{cases}1, & |t|<\pi \\ \frac{1}{2}, & |t|=\pi \\ 0, & |t|>\pi .\end{cases}
$$

Furthermore, one has

$$
\int_{\mathbb{R}} \operatorname{sinc}(u-s) \operatorname{sinc}(u-t) d u=\operatorname{sinc}(s-t) \quad(s, t \in \mathbb{R}),
$$

and, in particular,

$$
\int_{\mathbb{R}} \operatorname{sinc}(u-j) \operatorname{sinc}(u-k) d u=\left\{\begin{array}{ll}
1, & j=k, \\
0, & j \neq k
\end{array} \quad(j, k \in \mathbb{Z}) .\right.
$$


Proof Equation (2.7) follows by a simple integration, and (2.8) follows from (2.7) by the Fourier inversion formula. (For an elementary proof of (2.8) without using the inversion formula see [10, p. 13 f].) As to (2.9) one has by Proposition 1(a), (c), and by (2.7) and (2.8)

$$
\begin{aligned}
\int_{\mathbb{R}} \operatorname{sinc}(u-s) \operatorname{sinc}(u-t) d u & =\int_{\mathbb{R}}\left[\frac{1}{\sqrt{2 \pi}} \operatorname{rect}(\cdot) \mathrm{e}^{i \cdot s}\right]^{\wedge}(u) \operatorname{sinc}(u-t) d u \\
& =\int_{\mathbb{R}}\left[\frac{1}{\sqrt{2 \pi}} \operatorname{rect}(v) \mathrm{e}^{i v s}\right] \operatorname{sinc}(\cdot-t)^{\wedge}(v) d u \\
& =\int_{\mathbb{R}}\left[\frac{1}{\sqrt{2 \pi}} \operatorname{rect}(v) \mathrm{e}^{i v s}\right]\left[\frac{1}{\sqrt{2 \pi}} \operatorname{rect}(v) e^{-i v t}\right] d v \\
& =\frac{1}{2 \pi} \int_{\mathbb{R}} \operatorname{rect}(v) \mathrm{e}^{-i v(t-s)} d v=\frac{1}{\sqrt{2 \pi}} \operatorname{rect}^{\wedge}(t-s) \\
& =\operatorname{sinc}(t-s)=\operatorname{sinc}(s-t) .
\end{aligned}
$$

Finally, (2.10) follows from (2.9) noting that $\operatorname{sinc}(j-k)=\delta_{j, k}$.

We shall also need the following result for the approximate identities $g_{\rho}$ of GaußWeierstraß and $\chi_{\rho}$ of Fejér; see [20, Sec. 3.1.3, 3.1.2, 3.2].

Proposition 2 (a) For $f \in C(\mathbb{R})$, we have

$$
\lim _{\rho \rightarrow 0+} \frac{1}{\sqrt{2 \pi}} \int_{\mathbb{R}} f(u) g_{\rho}(t-u) d u=f(t)
$$

uniformly for $t \in \mathbb{R}$, where

$$
g_{\rho}(u):=\frac{1}{\sqrt{2 \rho}} \exp \left(\frac{-u^{2}}{4 \rho}\right) \quad(u \in \mathbb{R} ; \rho>0)
$$

is the Gaussian kernel, its Fourier transform being

$$
\widehat{g}_{\rho}(v)=e^{-\rho v^{2}} \quad(v \in \mathbb{R} ; \rho>0) .
$$

The same result is valid for $f \in L^{p}(\mathbb{R}), 1 \leq p<\infty$; the convergence in (2.11) holding now in $L^{p}(\mathbb{R})$-norm as well as a.e. on $\mathbb{R}$.

(b) The assertions of part (a) remain valid, if the Gaussian kernel is replaced by Fejér's kernel

$$
\chi_{\rho}(u):=\rho \sqrt{2 \pi} \operatorname{sinc}^{2}(\rho u) \quad(u \in \mathbb{R}, \rho>0)
$$

having Fourier transform

$$
\widehat{\chi}_{\rho}(v)=\left(1-\frac{|v|}{2 \pi \rho}\right)_{+} \quad(v \in \mathbb{R}, \rho>0),
$$

where $h_{+}(u):=h(u)$ if $h(u) \geq 0$, and $h_{+}(u)=0$ if $h(u)<0$. 
For $f:=\operatorname{sinc}(\cdot-u)$, we obtain from ASF the following sinc summation formula:

$$
\operatorname{sinc}(t-u)=\sum_{k \in \mathbb{Z}} \operatorname{sinc}(t-k) \operatorname{sinc}(u-k) .
$$

However, we need not invoke ASF for deriving (2.16). It is known that (2.16) is a simple consequence of the famous, classical cotangent expansion. For the latter, easy elementary proofs are known; see e.g. [43], [15, p. 448]

It follows from (2.16) that

$$
\sum_{k \in \mathbb{Z}} \operatorname{sinc}^{2}(t-k)=1 \quad(t \in \mathbb{R}) .
$$

The following lemma will be useful; for a proof see [40, p. 207].

Lemma 2 Let $f \in L^{p}(\mathbb{R}), 1<p<\infty$, and $\left(f_{k}\right)_{k \in \mathbb{N}}$ be a sequence in $L^{p}(\mathbb{R})$. If there exists a constant $M$ such that $\left\|f_{k}\right\|_{L^{p}(\mathbb{R})} \leq M$ for all $k \in \mathbb{N}$, and if $\lim _{k \rightarrow \infty} f_{k}(u)=$ $f(u)$ a.e. in $\mathbb{R}$, then for every $g \in L^{q}(\mathbb{R}), 1 / p+1 / q=1$,

$$
\lim _{k \rightarrow \infty} \int_{\mathbb{R}} f_{k}(u) g(u) d u=\int_{\mathbb{R}} f(u) g(u) d u .
$$

2.3 The sampling series $S_{w} f$

Concerning the convergence of the series

$$
\left(S_{w} f\right)(t):=\sum_{k \in \mathbb{Z}} f\left(\frac{k}{w}\right) \operatorname{sinc}(w t-k) \quad(t \in \mathbb{R}),
$$

occurring in (1.6), (1.7), we have

Lemma 3 Let $f \in S_{w}^{1}$ for some $w>0$. The series $\left(S_{w} f\right)(t)$ converges absolutely and uniformly on $\mathbb{R}$. Furthermore, $S_{w} f$ converges in $L^{p}(\mathbb{R}), 1<p<\infty$. In particular, $S_{w} f \in L^{p}(\mathbb{R})$, and

$$
\lim _{n \rightarrow \infty} \int_{\mathbb{R}}\left(S_{w, n} f\right)(u) \bar{g}(u) d u=\int_{\mathbb{R}}\left(S_{w} f\right)(u) \bar{g}(u) d u
$$

for all $g \in L^{q}(\mathbb{R}), 1 / p+1 / q=1$, where

$$
\left(S_{w, n} f\right)(t):=\sum_{|k| \leq n} f\left(\frac{k}{w}\right) \operatorname{sinc}(w t-k)
$$

denote the partial sums of $S_{w} f$. 
Proof For simplicity take $w=1$. For $0<n_{1}<n_{2}$ there holds

$$
\begin{aligned}
\left\|S_{1, n_{1}} f-S_{1, n_{1}} f\right\|_{L^{p}(\mathbb{R})} & \leq \sum_{n_{1}<|k| \leq n_{2}}|f(k)|\|\operatorname{sinc}(\cdot-k)\|_{L^{p}(\mathbb{R})} \\
& =\|\operatorname{sinc}\|_{L^{p}(\mathbb{R})} \sum_{n_{1}<|k| \leq n_{2}}|f(k)|,
\end{aligned}
$$

where the latter term tends to zero for $n_{1}, n_{2} \rightarrow \infty$ since $f \in S_{1}^{1}$. The same estimate holds for the space $C(\mathbb{R})$, giving the absolute and uniform convergence of $\left(S_{1, n} f\right)(t)$ to $\left(S_{1} f\right)(t)$. Further, the completeness of $L^{p}(\mathbb{R})$ and estimate (2.21) guarantee that $S_{1, n} f$ tends to some function $g \in L^{p}(\mathbb{R})$ which must be equal to the uniform limit $S_{1} f$.

Finally, by Hölder's inequality

$\left|\int_{\mathbb{R}}\left(S_{w, n} f\right)(u) \bar{g}(u) d u-\int_{\mathbb{R}}\left(S_{w} f\right)(u) \bar{g}(u) d u\right| \leq\left\|S_{w, n} f-S_{w} f\right\|_{L^{p}(\mathbb{R})}\|g\|_{L^{q}(\mathbb{R})}$,

which yields (2.19).

\subsection{Remarks on Poisson's summation formula}

As to the PSF, let $h(x):=\sqrt{2 \pi} w \sum_{k \in \mathbb{Z}}|f(x+2 k \pi w)|$; then

$$
\begin{aligned}
\int_{-\pi w}^{\pi w} h(x) d x & =\sqrt{2 \pi} w \sum_{k \in \mathbb{Z}} \int_{-\pi w}^{\pi w}|f(x+2 k \pi w)| d x \\
& =\sqrt{2 \pi} w \sum_{k \in \mathbb{Z}} \int_{(2 k-1) \pi w}^{(2 k+1) \pi w}|f(x)| d x=\sqrt{2 \pi} w \int_{\mathbb{R}}|f(x)| d x<\infty,
\end{aligned}
$$

showing that $h$ is integrable over the interval $[-\pi w, \pi w]$, and hence over any compact interval in view of the $2 \pi w$-periodicity of $h$.

Now one has for arbitrary $a, b \in \mathbb{R}$ that

$$
\int_{a}^{b}\left|\sqrt{2 \pi} w \sum_{k=m}^{n} f(x+2 k \pi w)\right| d x \leq \int_{a}^{b} h(x) d x<\infty \quad(m, n \in \mathbb{Z})
$$

and so it follows that the left-hand side of (1.10) converges a.e. on $\mathbb{R}$, and the convergence is dominated by the locally integrable function $h$.

When we wish to deduce PSF from any of the other formulae, it suffices to prove it for a dense subspace of $X \subset L^{1}(\mathbb{R})$ with $\widehat{g} \in S_{w}^{1}$ for all $g \in X$. Indeed, let $f \in L^{1}(\mathbb{R})$ with $\widehat{f} \in S_{w}^{1}$, and let $\left(f_{n}\right)_{n \in \mathbb{N}}$ be a sequence in $X$ with $\lim _{n \rightarrow \infty}\left\|f_{n}-f\right\|_{L^{1}(\mathbb{R})}=0$. Denoting $f^{*}(x):=\sqrt{2 \pi} w \sum_{k \in \mathbb{Z}} f(x+2 k \pi w), f_{n}^{*}(x):=\sqrt{2 \pi} w \sum_{k \in \mathbb{Z}} f_{n}(x+$ $2 k \pi w)$, then (cf. (2.23), (2.22)), 


$$
\int_{-\pi w}^{\pi w}\left|f_{n}^{*}(u)-f^{*}(u)\right| d u \leq\left\|f_{n}-f\right\|_{L^{1}(\mathbb{R})} \quad(n \in \mathbb{N}) .
$$

This yields that

$$
\lim _{n \rightarrow \infty} \widehat{f}_{n}^{*}(k)=\widehat{f}^{*}(k) \quad(k \in \mathbb{Z}),
$$

where $\widehat{f}_{n}^{*}(k)$ and $\widehat{f}^{*}(k)$ denote the trigonometric Fourier coefficients of the $2 \pi w$ periodic function $f_{n}^{*}$ and $f^{*}$, respectively.

On the other hand, PSF applied to the $f_{n}$ states that

$$
f_{n}^{*}(x)=\sum_{k \in \mathbb{Z}} \widehat{f_{n}}\left(\frac{k}{w}\right) e^{i k x / w} \quad \text { a.e. }
$$

meaning, in view of the uniform convergence of the series on the right, that $\widehat{f}_{n}(k / w)$, $k \in \mathbb{Z}$, are the Fourier coefficients of the $2 \pi w$-periodic function on the left. This implies that

$$
\widehat{f}^{*}(k)=\lim _{n \rightarrow \infty} \widehat{f}_{n}^{*}(k)=\lim _{n \rightarrow \infty} \widehat{f}_{n}\left(\frac{k}{w}\right)=\widehat{f}\left(\frac{k}{w}\right) \quad((k \in \mathbb{Z}),
$$

the last equality being valid in view of $\left|\widehat{f_{n}}(v)-\widehat{f}(v)\right| \leq\left\|f_{n}-f\right\|_{L^{1}(\mathbb{R})}$ for all $v \in \mathbb{R}$. Hence the Fourier coefficients of $f^{*}$ are given by $\widehat{f}^{*}(k)=\widehat{f}(k / w), k \in \mathbb{Z}$, i.e.

$$
f^{*}(x)=\sum_{k \in \mathbb{Z}} \widehat{f}\left(\frac{k}{w}\right) e^{i k x / w} \quad \text { a.e. }
$$

The equality a.e. here holds in view of $\widehat{f} \in S_{w}^{1}$, giving the uniform convergence of the Fourier series of $f^{*}$. This is PSF for all $f \in L^{1}(\mathbb{R})$ with $\widehat{f} \in S_{w}^{1}$.

\section{Equivalence of the decomposition formula and four summation formulae}

In this section we shall deduce the basic new ARKF theorem for $L^{2}(\mathbb{R})$-function by means of the ASF. Even more so, the ASF itself is a consequence of ARKF. Thus the two are equivalent. On top, three other basic equivalences of this paper are also established in this section.

When we want to deduce one of the formulae GPDF, ASF or ARKF, it suffices to carry it out for $w=1$. Indeed, the general formula is obtained from that special case by replacing $f$ by $f(\cdot / w), t$ by $w t$ and noting that

$$
\left[\left(R_{1} f\right)\left(\frac{\dot{w}}{w}\right)\right](w t)=\left(R_{w} f\right)(t)
$$




\subsection{GPDF and ASF}

But first to the equivalence of GPDF with ASF. The proof of the implication $\mathrm{GPDF} \Rightarrow \mathrm{ASF}$ is essentially that of Thm 1.3 in [18]. We present it here again for convenience.

Proof of $G P D F \Rightarrow A S F$ for $f \in F^{2} \cap S_{w}^{1}$. In regard to the proof which follows, it is appropriate to rewrite (1.1) as

$$
\begin{aligned}
\int_{\mathbb{R}} f(u) \bar{g}(u) d u= & \frac{1}{w} \sum_{k \in \mathbb{Z}} f\left(\frac{k}{w}\right) \frac{1}{\sqrt{2 \pi}} \int_{|v| \leq \pi w} \widehat{\bar{g}}(v) e^{i k v / w} d v \\
& +\int_{\mathbb{R}}\left(R_{w} f\right)(u) \bar{g}(u) d u .
\end{aligned}
$$

Indeed, one has in view of the Fourier inversion formula (2.6) for $g$

$$
\begin{aligned}
& \frac{1}{w} \sum_{k \in \mathbb{Z}} f\left(\frac{k}{w}\right) \bar{g}\left(\frac{k}{w}\right)-\frac{1}{w} \sum_{k \in \mathbb{Z}} f\left(\frac{k}{w}\right) \frac{1}{\sqrt{2 \pi}} \int_{|v| \geq \pi w} \widehat{\bar{g}}(v) \mathrm{e}^{i k v / w} d v \\
& \quad=\frac{1}{w} \sum_{k \in \mathbb{Z}} f\left(\frac{k}{w}\right) \frac{1}{\sqrt{2 \pi}}\left\{\int_{-\infty}^{\infty} \widehat{\bar{g}}(v) \mathrm{e}^{i k v / w} d v-\int_{|v| \geq \pi w} \widehat{\bar{g}}(v) \mathrm{e}^{i k v / w} d v\right\} .
\end{aligned}
$$

A substitution of this identity into (1.1) yields (3.1).

Now we restrict the matter to $w=1$ and apply the GPDF in the form (3.1) to $f \in F^{2} \cap S_{1}^{1}$ and $g=(2 \pi)^{-1 / 2} g_{\rho}(t-\cdot) \in F^{2}$ of $(2.12)$. Noting that $\left.\widehat{g_{\rho}(t-} \cdot\right)(v)=$ $e^{-\rho v^{2}} e^{-i t v}$ by (2.1) and (2.13), we obtain

$$
\begin{aligned}
& \frac{1}{\sqrt{2 \pi}} \int_{\mathbb{R}} f(u) g_{\rho}(t-u) d u=\sum_{k \in \mathbb{Z}} f(k) \frac{1}{2 \pi} \int_{|v| \leq \pi} e^{-\rho v^{2}} \mathrm{e}^{i v(k-t)} d v \\
& \quad+\frac{1}{\sqrt{2 \pi}} \int_{\mathbb{R}}\left(R_{1} f\right)(u) g_{\rho}(t-u) d u .
\end{aligned}
$$

In this equation, we let $\rho \rightarrow 0+$. Since $f$ and $R_{1} f$ belong to $C(\mathbb{R})$, and since the infinite series is uniformly convergent in view of $f \in S_{1}^{1}$, Proposition 2 yields

$$
f(t)=\sum_{k \in \mathbb{Z}} f(k) \frac{1}{2 \pi} \int_{|v| \leq \pi} \mathrm{e}^{i v(k-t)} d v+\left(R_{1} f\right)(t) \quad(t \in \mathbb{R}) .
$$

This is ASF, because the integral equals $2 \pi \operatorname{sinc}(t-k)$. The absolute and uniform convergence of the series $\left(S_{1} f\right)(t)$ follows from Lemma 3 .

The proof of the converse direction $\mathrm{ASF} \Rightarrow \mathrm{GPDF}$ is similar to the corresponding one in [18] for non-uniform sampling. 
Proof of $A S F \Rightarrow G P D F$ for $f \in F^{2} \cap S_{w}^{1}, g \in F^{2}$. Let $w=1$, multiply ASF by $\bar{g}$ and integrate over $\mathbb{R}$. Then

$$
\int_{\mathbb{R}} f(u) \bar{g}(u) d u=\int_{\mathbb{R}}\left(S_{1} f\right)(u) \bar{g}(u) d u+\int_{\mathbb{R}}\left(R_{1} f\right)(u) \bar{g}(u) d u .
$$

Since $f \in L^{2}(\mathbb{R})$ by assumption, we also have $S_{1} f \in L^{2}(\mathbb{R})$ in view of Lemma 3 , and hence $R_{1} f=f-S_{w} f \in L^{2}(\mathbb{R})$. Thus all integrals in (3.2) exists. For the first term on the right-hand side of (3.2) one obtains

$$
\int_{\mathbb{R}}\left(S_{1} f\right)(u) \bar{g}(u) d u=\sum_{k \in \mathbb{Z}} f(k) \int_{\mathbb{R}} \bar{g}(u) \operatorname{sinc}(u-k) d u,
$$

the interchange of integration and summation being justified, since $(f(k)) \in l^{1}(\mathbb{Z})$ and the integrals on the right-hand side are bounded by $\|g\|_{L^{2}(\mathbb{R})} \cdot\|\operatorname{sinc}\|_{L^{2}(\mathbb{R})}$. Now, using the convolution theorem (2.4), formula (2.8), and the Fourier inversion formula (2.6), we can rewrite the latter integral as

$$
\begin{gathered}
\int_{\mathbb{R}} \bar{g}(u) \operatorname{sinc}(u-k) d u=\sqrt{2 \pi}(\bar{g} * \operatorname{sinc})(k)=\frac{1}{\sqrt{2 \pi}} \int_{\mathbb{R}} \widehat{\bar{g}}(v) \operatorname{rect}(v) e^{i k v} d v \\
=\frac{1}{\sqrt{2 \pi}}\left\{\int_{\mathbb{R}}-\int_{|v| \geq \pi}\right\} \widehat{\bar{g}}(v) e^{i k v} d v=\bar{g}(k)-\frac{1}{\sqrt{2 \pi}} \int_{|v| \geq \pi} \widehat{\bar{g}}(v) e^{i k v} d v
\end{gathered}
$$

Inserting this into (3.3), and then the resulting equation into (3.2) yields the assertion (1.1) for $w=1$.

Thus we have established the equivalence $\mathrm{ASF} \Leftrightarrow \mathrm{GPDF}$.

\subsection{ASF and ARKF}

Now to the basic equivalence ASF $\Leftrightarrow$ ARKF mentioned above.

Proof of ASF $\Rightarrow A R K F$ for $f \in F^{2} \cap S_{w}^{1}, g \in F^{2}$. The fact that $R_{w} f=f-S_{w} f \in$ $L^{2}(\mathbb{R})$ follows again from Lemma 3. Now, let $w=1$ and let $f$ be as in ARKF. We consider ASF (1.6), replace $t$ by $u$, multiply both sides by $\operatorname{sinc}(t-u)$ and integrate with respect to $u$. This gives

$$
\begin{aligned}
& \int_{\mathbb{R}} f(u) \operatorname{sinc}(t-u) d u=\int_{\mathbb{R}} \sum_{k \in \mathbb{Z}} f(k) \operatorname{sinc}(u-k) \operatorname{sinc}(t-u) d u \\
& +\int_{\mathbb{R}}\left(R_{1} f\right)(u) \operatorname{sinc}(t-u) d u .
\end{aligned}
$$

By Lemma 3 we can interchange integration and summation on the right-hand side and we obtain by (2.9) 


$$
\begin{aligned}
& \int_{\mathbb{R}} \sum_{k \in \mathbb{Z}} f(k) \operatorname{sinc}(u-k) \operatorname{sinc}(t-u) d u \\
& =\sum_{k \in \mathbb{Z}} f(k) \int_{\mathbb{R}} \operatorname{sinc}(u-k) \operatorname{sinc}(t-u) d u=\sum_{k \in \mathbb{Z}} f(k) \operatorname{sinc}(t-k) .
\end{aligned}
$$

Applying ASF once more, the latter series can be replaced by $f(t)-\left(R_{1} f\right)(t)$, and hence

$$
\int_{\mathbb{R}} \sum_{k \in \mathbb{Z}} f(k) \operatorname{sinc}(u-k) \operatorname{sinc}(t-u) d u=f(t)-\left(R_{1} f\right)(t) .
$$

Inserting this into (3.4) yields the ARKF in the form (1.9).

Proof of $A R K F \Rightarrow A S F$ for $f \in F^{2} \cap S_{1}^{1}$. It suffices to show that

$$
\int_{\mathbb{R}}\left[f(u)-\left(R_{1} f\right)(u)\right] \operatorname{sinc}(t-u) d u=\sum_{k \in \mathbb{Z}} f(k) \operatorname{sinc}(t-k),
$$

since the integral equals $f(t)-\left(R_{1} f\right)(t)$. For this purpose, we shall use the sinc summation formula (2.16) on the left-hand side and interchange integration and summation. For justifying the interchange, we want to employ Lemma 2. We have to verify that the hypotheses of that result are satisfied in the present situation.

Clearly, $f-R_{1} f$ and $\operatorname{sinc}(\cdot-t)$ belong to $L^{2}(\mathbb{R})$. Next, setting

$$
\operatorname{sinc}_{N}(t-u):=\sum_{|k| \leq N} \operatorname{sinc}(u-k) \operatorname{sinc}(t-k),
$$

we have $\lim _{N \rightarrow \infty} \operatorname{sinc}_{N}(t-u)=\operatorname{sinc}(t-u)$. Furthermore, by employing (2.9) and (2.17), we find that

$$
\begin{aligned}
\| & \operatorname{sinc}_{N}(t-\cdot) \|_{L^{2}(\mathbb{R})}^{2} \\
& =\sum_{|k| \leq N} \sum_{|l| \leq N}\left\{\int_{\mathbb{R}} \operatorname{sinc}(u-k) \operatorname{sinc}(u-l) \mathrm{d} u\right\} \operatorname{sinc}(t-k) \operatorname{sinc}(t-l) \\
& =\sum_{|k| \leq N} \sum_{|l| \leq N} \delta_{k, l} \cdot \operatorname{sinc}(t-k) \operatorname{sinc}(t-l) \\
& =\sum_{|k| \leq N} \operatorname{sinc}^{2}(t-k) \leq \sum_{k \in \mathbb{Z}} \operatorname{sinc}^{2}(t-k)=1,
\end{aligned}
$$

where Kronecker's delta has been used. Now Lemma 2 allows us to conclude that

$$
\begin{aligned}
& \int_{\mathbb{R}}\left[f(u)-R_{1} f(u)\right] \operatorname{sinc}(t-u) \mathrm{d} u \\
& =\lim _{N \rightarrow \infty} \int_{\mathbb{R}}\left[f(u)-R_{1} f(u)\right] \operatorname{sinc}_{N}(t-u) \mathrm{d} u \\
& =\lim _{N \rightarrow \infty} \sum_{|k| \leq N}\left\{\int_{\mathbb{R}}\left[f(u)-R_{1} f(u)\right] \operatorname{sinc}(u-k) \mathrm{d} u\right\} \operatorname{sinc}(t-k) .
\end{aligned}
$$


By ARKF for $t=k$, the expression in braces is equal to $f(k)-R_{1} f(k)$. Since $R_{1} f$ vanishes at the integers, we see that (3.5) holds. The statement regarding the convergence of the series is verified as in the proof of GPDF $\Rightarrow$ ASF.

The equivalence assertion $\mathrm{ASF} \Leftrightarrow \mathrm{ARKF}$, just established, is another new result of this paper.

\subsection{PSF and PDPS}

The following concerns the final grouping of this section.

Proof of $P S F \Rightarrow P D P S$ It suffices again to consider the case $w=1$. First we assume that $g \in F^{2}$ only and define

$$
\bar{g}_{1}(u):=\frac{1}{\sqrt{2 \pi}} \int_{-\sigma}^{\sigma} \widehat{\bar{g}}(v) e^{i u v} d v \quad \text { and } \quad \phi(u):=\left(f \bar{g}_{1}\right) \widehat{(}(-u)
$$

which implies that $\widehat{\phi}=f \bar{g}_{1}$. Then $\widehat{\phi} \in L^{1}(\mathbb{R})$ since it is a product of two functions from $L^{2}(\mathbb{R})$, and $\widehat{\phi} \in S_{1}^{1}$ since $f \in S_{1}^{1}$ and $\bar{g}_{1}$ is bounded. Furthermore, $\phi \in C(\mathbb{R})$ since $\phi(-\cdot)$ is the Fourier transform of a function from $L^{1}(\mathbb{R})$. Now one has by Proposition 1 (a), (c) that

$$
\begin{aligned}
\phi(-\xi)=\left(f \bar{g}_{1}\right) \widehat{f}(\xi) & =\frac{1}{\sqrt{2 \pi}} \int_{\mathbb{R}} f(v) \bar{g}_{1}(v) e^{-i v \xi} d v \\
& =\frac{1}{\sqrt{2 \pi}} \int_{\mathbb{R}}\left[f(\cdot) e^{-i \xi \cdot}\right] \widehat{\widehat{f}}(-v) \bar{g}_{1}(v) d v \\
& =\frac{1}{\sqrt{2 \pi}} \int_{\mathbb{R}} \widehat{f}(\xi-v) \widehat{\bar{g}}_{1}(v) d v .
\end{aligned}
$$

Hence $\phi(-\cdot)$ is the convolution of $\widehat{f} \in L^{1}(\mathbb{R})$ and $\widehat{\bar{g}}_{1} \in L^{1}(\mathbb{R})$, and so it follows by Proposition $1 \mathrm{~b}$ ) that $\phi \in L^{1}(\mathbb{R})$. Altogether we have shown that $\phi$ satisfies the hypotheses of PSF for $w=1$, and so

$$
\sqrt{2 \pi} \sum_{k \in \mathbb{Z}} \phi(x+2 k \pi)=\sum_{k \in \mathbb{Z}} \widehat{\phi}(k) e^{i k x}=\sum_{k \in \mathbb{Z}} f(k) \bar{g}_{1}(k) e^{i k x} \text { a.e. }
$$

Employing (3.6) and noting that $\widehat{\bar{g}}_{1}(v)=\overline{\widehat{g}}_{1}(-v)=\overline{\widehat{g}}(-v) \operatorname{rect}(v \pi / \sigma)$, we find that

$$
\phi(\xi)=\left(f \bar{g}_{1}\right) \widehat{f}(-\xi)=\frac{1}{\sqrt{2 \pi}} \int_{-\sigma}^{\sigma} \widehat{f}(v-\xi) \widehat{g}(v) d v
$$

Substituting this into the left-hand side of (3.7) yields

$$
\sum_{k \in \mathbb{Z}} \int_{-\sigma}^{\sigma} \widehat{f}(v-x-2 k \pi) \widehat{g}(v) d v=\sum_{k \in \mathbb{Z}} f(k) \bar{g}_{1}(k) e^{i k x} \quad \text { a.e. }
$$


Using the Fourier inversion formula to obtain

$$
\bar{g}_{1}(u)=\bar{g}(u)-\frac{1}{\sqrt{2 \pi}} \int_{|v| \geq \sigma} \widehat{\bar{g}}(v) e^{i u v} d v
$$

for eliminating $\bar{g}_{1}$ on the right-hand side of (3.8), we arrive at

$$
\begin{aligned}
& \sum_{k \in \mathbb{Z}} \int_{-\sigma}^{\sigma} \widehat{f}(v-x-2 k \pi) \widehat{\widehat{g}}(v) d v \\
& \quad=\sum_{k \in \mathbb{Z}} f(k) \bar{g}(k) e^{i k x}-\sum_{k \in \mathbb{Z}} f(k) \frac{1}{\sqrt{2 \pi}} \int_{|v| \geq \sigma} \widehat{\widehat{g}}(v) e^{i k(v+x)} d v \text { a.e. }
\end{aligned}
$$

The desired formula is obtained by replacing $k$ by $-k$ on the left-hand side.

When $g \in F^{1}$, then $\bar{g} \in C(\mathbb{R})$. Since also $f \in S_{1}^{1}$ and $\bar{g} \in L^{1}(\mathbb{R})$, we observe that the two series on the right-hand side of (3.9) depend continuously on $x$.

As to the left-hand side, choose $n \in \mathbb{N}$ such that $n \pi \geq \sigma$, then,

$$
\begin{aligned}
\left|\sum_{k \in \mathbb{Z}} \int_{-\sigma}^{\sigma} \widehat{f}(v-2 k \pi) \widehat{g}(v) d v\right| & \leq\|\widehat{g}\|_{L^{\infty}(\mathbb{R})} \sum_{k \in \mathbb{Z}} \int_{-n \pi}^{n \pi}|\widehat{f}(v-2 k \pi)| d v \\
& =\|\widehat{g}\|_{L^{\infty}(\mathbb{R})} \int_{-n \pi}^{n \pi} \sum_{k \in \mathbb{Z}}|\widehat{f}(v-2 k \pi)| d v .
\end{aligned}
$$

Here and in the next displayed formula the interchange of summation and integration is justified by Beppo Levi's theorem.

Since the integrand in the last line of (3.10) is a $2 \pi$-periodic function, the integral equals $n$-times the integral from $-\pi$ to $\pi$, and hence

$$
\begin{aligned}
& \left|\sum_{k \in \mathbb{Z}} \int_{-\sigma}^{\sigma} \widehat{f}(v-2 k \pi) \widehat{\widehat{g}}(v) d v\right| \leq n\|\widehat{g}\|_{L^{\infty}(\mathbb{R})} \int_{-\pi}^{\pi} \sum_{k \in \mathbb{Z}}|\widehat{f}(v-2 k \pi)| d v \\
& \quad=n\|\widehat{g}\|_{L^{\infty}(\mathbb{R})} \sum_{k \in \mathbb{Z}} \int_{(-2 k-1) \pi}^{(-2 k+1) \pi}|\widehat{f}(v)| d v=n\|\widehat{g}\|_{L^{\infty}(\mathbb{R})} \int_{-\infty}^{\infty}|\widehat{f}(v)| d v .
\end{aligned}
$$

This inequality, with $\widehat{f}(\cdot)$ replaced by $\widehat{f}(\cdot-x)-\widehat{f}(\cdot-y)$, yields

$$
\begin{aligned}
& \left|\sum_{k \in \mathbb{Z}} \int_{-\sigma}^{\sigma} \widehat{f}(v-x-2 k \pi) \widehat{\widehat{g}}(v) d v-\sum_{k \in \mathbb{Z}} \int_{-\sigma}^{\sigma} \widehat{f}(v-y-2 k \pi) \widehat{g}(v) d v\right| \\
& \quad \leq n\|\widehat{g}\|_{L^{\infty}(\mathbb{R})} \int_{-\infty}^{\infty}|\widehat{f}(v-x)-\widehat{f}(v-y)| d v,
\end{aligned}
$$

and since the right side of this inequality tends to zero for $y \rightarrow x$ by the continuity in the mean of $\widehat{f} \in L^{1}(\mathbb{R})$, we have shown that the left-hand side of (3.9) is a continuous 
function of $x \in \mathbb{R}$. Thus, both sides being continuous, (3.9) must hold for all $x \in \mathbb{R}$.

Proof of PDPS $\Rightarrow$ PSF for $f \in L^{1}(\mathbb{R})$ with $\widehat{f} \in S_{1}^{1}$. First assume $f \in F^{1}(\mathbb{R})$ with $\widehat{f} \in S_{1}^{1}$. We apply PDPS with $\sigma=\pi, f$ replaced by $\widehat{f} \in F^{1} \cap S_{1}^{1} \subset F^{2} \cap S_{1}^{1}$, and $g(t)$ replaced by $\widehat{g}_{\rho}(t) e^{-i x t} \in F^{1}$ of (2.12), (2.13). Noting that $g_{\rho}, \widehat{g}_{\rho}$ are real and even, (2.6), and

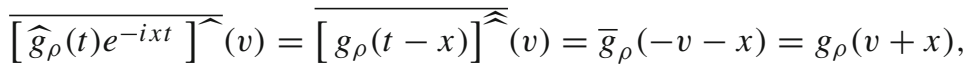

$$
\begin{aligned}
& {\left[\overline{\widehat{g}_{\rho}(t) e^{-i x t}}\right] \widehat{(v)}=\left[\widehat{g}_{\rho}(v) e^{i x t}\right] \widehat{(v)}=\left[g_{\rho}(t+x)\right] \widehat{\widehat{(}}(v)=g_{\rho}(x-v) \text {, }}
\end{aligned}
$$

we obtain for each $x \in \mathbb{R}$,

$$
\begin{aligned}
\sum_{k \in \mathbb{Z}} \widehat{f}(k) \widehat{g}_{\rho}(k) e^{i x k}= & \sum_{k \in \mathbb{Z}} \int_{-\pi}^{\pi} \widehat{\widehat{f}}(v+2 k \pi) g_{\rho}(v+x) d v \\
& +\sum_{k \in \mathbb{Z}} \widehat{f}(k) \frac{1}{\sqrt{2 \pi}} \int_{|v| \geq \pi} g_{\rho}(x-v) \mathrm{e}^{i k v} d v .
\end{aligned}
$$

Now let $\rho \rightarrow 0+$. Since $\lim _{\rho \rightarrow 0+} \widehat{g}_{\rho}(k)=1$ by $(2.13)$, it follows that

$$
\lim _{\rho \rightarrow 0+} \sum_{k \in \mathbb{Z}} \widehat{f}(k) \widehat{g}_{\rho}(k) e^{i x k}=\sum_{k \in \mathbb{Z}} \widehat{f}(k) e^{i x k} \quad(x \in \mathbb{R}),
$$

where the interchange of summation and integration is allowed since the series is uniformly convergent with respect to $\rho>0$ because of $\{\widehat{f}(k)\} \in \ell^{1}(\mathbb{Z})$ and $0<$ $\widehat{g}_{\rho}(k) \leq 1$ for all $k \in \mathbb{Z}$ and $\rho>0$.

As to the right-hand side of (3.11), the first term can be rewritten as (cf. (2.6)),

$$
\begin{aligned}
\sum_{k \in \mathbb{Z}} \int_{-\pi}^{\pi} \widehat{\widehat{f}}(v+2 k \pi) g_{\rho}(v+x) d v & =\sum_{k \in \mathbb{Z}} \int_{-\pi}^{\pi} f(v-2 k \pi) g_{\rho}(x-v) d v \\
& =\int_{\mathbb{R}}\left\{\sum_{k \in \mathbb{Z}} f(v+2 k \pi)\right\} \operatorname{rect}(v) g_{\rho}(x-v) d v
\end{aligned}
$$

where the interchange of summation and integration is justified in view of the dominated convergence of the series. An application of Proposition 2 shows that the latter integral tends to $\sqrt{2 \pi} \sum_{k \in \mathbb{Z}} f(v+2 k \pi)$ a.e. on $(-\pi, \pi)$ for $\rho \rightarrow 0+$.

The integral in the second term on the right-hand side of (3.11) can be rewritten as

$$
\frac{1}{\sqrt{2 \pi}} \int_{\mathbb{R}} g_{\rho}(x-v) \mathrm{e}^{i k v}\{1-\operatorname{rect}(v)\} d v,
$$

which tends to zero for $\rho \rightarrow 0+$ a. e. on $(-\pi, \pi)$ by Proposition 2 . Hence we obtain

$$
\lim _{\rho \rightarrow 0+} \sum_{k \in \mathbb{Z}} \widehat{f}(k) \frac{1}{\sqrt{2 \pi}} \int_{|v| \geq \pi} g_{\rho}(v-x) \mathrm{e}^{i k v} d v=0 \quad(\text { a.e. on }(-\pi, \pi)),
$$


noting again that $\{\widehat{f}(k)\} \in \ell^{1}(\mathbb{Z})$.

Altogether it follows from (3.11) for $\rho \rightarrow 0+$,

$$
\sum_{k \in \mathbb{Z}} \widehat{f}(k) e^{i x k}=\sqrt{2 \pi} \sum_{k \in \mathbb{Z}} f(v+2 k \pi) \quad(\text { a.e. on }(-\pi, \pi))
$$

Since both sides of (3.12) define a $2 \pi$-periodic function, equation (3.12) holds even a.e. on $\mathbb{R}$, which is PSF for the space $\left\{f \in F^{1} ; \widehat{f} \in S_{1}^{1}\right\} \subset L^{1}(\mathbb{R})$.

In order to extend PSF to the whole of $L^{1}(\mathbb{R})$ with $\widehat{f} \in S_{1}^{1}$, we have only to note the remarks on PSF in Sec. 2.4, since $\left\{f \in F^{1} ; \widehat{f} \in S_{1}^{1}\right\}$ is dense in $L^{1}(\mathbb{R})$. For example, the convolution $f_{\rho}:=f * g_{\rho}$ of $f \in L^{1}(\mathbb{R})$ with $g_{\rho}$ of (2.12) belongs to $F^{1}, \widehat{f}_{\rho} \in S_{1}^{1}$ for all $\rho>0$, and $\lim _{\rho \rightarrow \infty} f_{\rho}=f$ in $L^{1}(\mathbb{R})$-norm (Proposition 2).

The equivalence PSF $\Leftrightarrow$ GPDF was established in [18] with the PSF-version

$$
\sqrt{2 \pi} \sum_{k \in \mathbb{Z}} \widehat{f}(x+2 k \pi w)=\sum_{k \in \mathbb{Z}} f\left(\frac{k}{w}\right) e^{i k x / w} \quad(x \in \mathbb{R}) .
$$

whereby the roles of $f$ and $\widehat{f}$ have been exchanged, under the additional assumption $\widehat{f} \in B V(\mathbb{R})$.

The present proof is modeled on that of [18], but the lack of the BV-assumption in our case causes some additional arguments.

\subsection{PSF and ASF}

As to the equivalence of PSF and ASF, it was already established in [23,24], however with different versions of PSF.

Proof of $P S F \Rightarrow A S F$ for $f \in F^{1} \cap S_{1}^{1}$. We again apply PSF (1.10) to the function $\widehat{f} \in F^{1}$, noting that $(\widehat{\hat{f}}(k))=(f(-k)) \in \ell^{1}(\mathbb{Z})$, giving

$$
\sqrt{2 \pi} \sum_{k \in \mathbb{Z}} \widehat{f}(v+2 k \pi)=\sum_{k \in \mathbb{Z}} f(k) e^{-i k v} \quad \text { a.e. }
$$

Hence, recalling (2.7),

$$
\begin{aligned}
& \frac{1}{\sqrt{2 \pi}} \int_{-\pi}^{\pi} \sum_{k \in \mathbb{Z}} \widehat{f}(v+2 k \pi) d v=\frac{1}{2 \pi} \int_{-\pi}^{\pi} \sum_{k \in \mathbb{Z}} f(k) e^{-i k v} e^{i v t} d v \\
& =\frac{1}{2 \pi} \sum_{k \in \mathbb{Z}} f(k) \int_{-\pi}^{\pi} e^{-i k v} e^{i v t} d v=\frac{1}{2 \pi} \sum_{k \in \mathbb{Z}} f(k) \int_{-\infty}^{\infty} \operatorname{rect}(v) e^{-i k v} e^{i v t} d v \\
& =\sum_{k \in \mathbb{Z}} f(k) \operatorname{sinc}(t-k)=\left(S_{1} f\right)(t) \quad(t \in \mathbb{R}),
\end{aligned}
$$

the interchange of summation and integration being justified in view of the absolute and uniform convergence of the series involved. 
On the other hand, we have

$$
\int_{-\pi}^{\pi} \sum_{k \in \mathbb{Z}} \widehat{f}(v+2 k \pi) e^{i v t} d v=\sum_{k \in \mathbb{Z}} \int_{(2 k-1) \pi}^{(2 k+1) \pi} \widehat{f}(v) e^{i t(v-2 k \pi)} d v \quad(t \in \mathbb{R}),
$$

yielding

$$
\left(S_{1} f\right)(t)=\frac{1}{\sqrt{2 \pi}} \sum_{k \in \mathbb{Z}} e^{-i 2 k \pi t} \int_{(2 k-1) \pi}^{(2 k+1) \pi} \widehat{f}(v) e^{i v t} d v \quad(t \in \mathbb{R}) .
$$

But by the Fourier inversion integral (2.6),

$$
f(t)=\frac{1}{\sqrt{2 \pi}} \int_{\mathbb{R}} \widehat{f}(v) e^{i v t} d v=\frac{1}{\sqrt{2 \pi}} \sum_{k \in \mathbb{Z}} \int_{(2 k-1) \pi}^{(2 k+1) \pi} \widehat{f}(v) e^{i v t} d v \quad(t \in \mathbb{R}) .
$$

Subtracting (3.13) from (3.14) yields $f(t)-\left(S_{1} f\right)(t)=\left(R_{1} f\right)(t)$, thus the approximate sampling theorem.

Proof of $A S F \Rightarrow P S F$ Noting (3.14) it follows immediately from ASF with remainder (1.2) that for $f \in F^{2}$ and $w=1$

$$
\sum_{k \in \mathbb{Z}} f(k) \operatorname{sinc}(t-k)=\frac{1}{\sqrt{2 \pi}} \sum_{k \in \mathbb{Z}} e^{-i 2 k \pi t} \int_{(2 k-1) \pi}^{(2 k+1) \pi} \widehat{f}(v) e^{i v t} d v \quad(t \in \mathbb{R}) .
$$

Using (2.5) and (2.7) one can rewrite the terms on the right-hand side as a convolution product, namely

$$
\begin{aligned}
& \frac{e^{-i 2 k \pi t}}{\sqrt{2 \pi}} \int_{(2 k-1) \pi}^{(2 k+1) \pi} \widehat{f}(v) e^{i t v} d v=\frac{e^{-i 2 k \pi t}}{\sqrt{2 \pi}} \int_{\mathbb{R}} \widehat{f}(v) \operatorname{rect}(v-2 k \pi) e^{i t v} d v \\
& =\int_{\mathbb{R}} f(u) e^{-i 2 k \pi u} \operatorname{sinc}(t-u) d u=\sqrt{2 \pi}\left[\left(f(\cdot) e^{-i 2 k \pi \cdot}\right) * \operatorname{sinc}(\cdot)\right](t) \quad(t \in \mathbb{R}) .
\end{aligned}
$$

For the following, we first assume $f \in \widehat{B}_{\sigma}^{1}$ for some $\sigma>0$. Then the terms on the right of (3.15) vanish for $|k|>\sigma_{0}:=(\sigma / \pi+1) / 2$, and (3.15) can be rewritten as

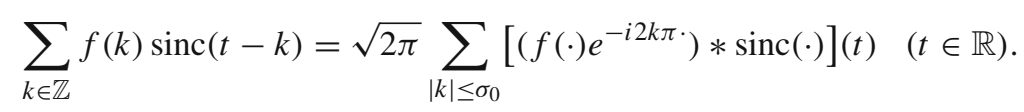

Now, taking the $L^{2}$-Fourier transform of this equation, noting that the series on the left converges with respect to $L^{2}(\mathbb{R})$-norm (Lemma 3), Proposition 1 and (2.8), we obtain

$$
\frac{1}{\sqrt{2 \pi}} \sum_{k \in \mathbb{Z}} f(k) \operatorname{rect}(v) e^{i k v}=\sum_{|k| \leq \sigma_{0}} \widehat{f}(v+2 k \pi) \operatorname{rect}(v) \text { a.e. }
$$


Since $f \in \widehat{B}_{\sigma}^{1}, \widehat{f}$ can be regarded as the $L^{1}$-Fourier transform, which is continuous. Then (3.16) holds even for every $v$ in a neighbourhood of the origin, since all functions involved are continuous there. So one may take $v=0$ to obtain

$$
\frac{1}{\sqrt{2 \pi}} \sum_{k \in \mathbb{Z}} f(k)=\sum_{|k| \leq \sigma_{0}} \widehat{f}(2 k \pi)
$$

Replacing now $f(\cdot)$ by $2 \pi f(x+2 \pi \cdot)$, and noting Proposition 1 (a) gives PSF.

It remains to remove the restriction $f \in \widehat{B}_{\sigma}^{1}$ for some $\sigma>0$. According to Sect. 2.4 it suffices to show that $\bigcup_{\sigma>0} \widehat{B}_{\sigma}^{1}$ is dense in $L^{1}(\mathbb{R})$. To see this, one may take $f * \chi_{\rho}, \rho>$ 0 , with $f \in L^{1}(\mathbb{R})$ and $\chi_{\rho}$ of (2.14). This convolution belongs to $\widehat{B}_{2 \pi \rho}^{1}$, noting (2.15) and Proposition 1 (b), and $\lim _{\rho \rightarrow \infty} f * \chi_{\rho}=f$ in $L^{1}(\mathbb{R})$-norm (Proposition 2 (b).

\section{PSF and the functional equation for $\zeta(s)$}

First some remarks concerning the $\zeta$-function. Since the series (1.15) is locally uniformly convergent in the half-plane $\sigma>1, \zeta(s)$ is analytic there. To extend it to a function which is analytic in the whole complex plane $\mathbb{C}$ except $s=1$, one can employ the representation

$$
\zeta(s)=s \int_{1}^{\infty} u^{-s-1}\left(\mu(u)+\frac{1}{2}\right) d u+\frac{1}{s-1}+\frac{1}{2}
$$

with the measure $\mu(u):=\lfloor u\rfloor-u$, where $\lfloor u\rfloor$ denotes the largest integer less than or equal to $u$; see Titchmarsh [82, (2.1.4)].

Noting that $\left|\int_{1}^{u}\left(\mu(t)+\frac{1}{2}\right) d t\right|$ is bounded by $\frac{1}{8}$ on $\mathbb{R}$, one can easily show by partial integration that the integral is locally uniformly convergent in the half-plane $\sigma>-1$. Hence (4.1) defines an extension of (1.15) to a function which is analytic for $\sigma>-1$ except for the point $s=1$, where there is a simple pole with residue 1 . This function can be continued further to a function which is analytic in the whole complex plane except for $s=1$.

From (4.1) one can also deduce by partial integration the estimate

$$
|\zeta(s)| \leq|s|+\frac{1}{|s-1|}+\frac{1}{2} \quad(s=\sigma+i \tau \in \mathbb{C} \backslash\{1\}, \sigma>-1) .
$$

In the strip $0<\sigma<1$ the representation (4.1) can be rewritten as (see Tichmarsh $(2.1 .5))$

$$
\zeta(s)=s \int_{0}^{\infty} u^{-s-1} \mu(u) d u \quad(s=\sigma+i \tau, 0<\tau<1),
$$

noting that

$$
\int_{0}^{1} u^{-s-1} \mu(u) d u=\int_{0}^{1} u^{-s} d u=\frac{1}{s-1}
$$


Now we turn to the equivalence PSF $\Leftrightarrow$ FERZ.

Proof of $P S F \Rightarrow F E R Z$ If $f$ belongs to the Schwartz space $\mathcal{S}$ of rapidly decreasing functions, the PSF (1.10), in its simplest form for $w=1, x=0$, reads

$$
\sqrt{2 \pi} \sum_{k \in \mathbb{Z}} f(2 k \pi)=\sum_{k \in \mathbb{Z}} \widehat{f}(k) .
$$

In fact, under the given assumption $f \in \mathcal{S}$, Eq. (1.10) holds for all $x \in \mathbb{R}$, since both series are uniformly convergent, at least on compact subsets of $\mathbb{R}$, and hence define continuous functions.

If $f$ is even, so will be $\widehat{f}$, and PSF turns into

$$
\sqrt{2 \pi}\left\{\sum_{k=1}^{\infty} f(2 \pi k)-\int_{0}^{\infty} f(2 \pi u) d u\right\}=\int_{0}^{\infty} \widehat{f}(v) d v-\sum_{k=1}^{\infty} \widehat{f}(k),
$$

noting that $\frac{1}{2} \widehat{f}(0)=(1 / \sqrt{2 \pi}) \int_{0}^{\infty} f(x) d x=\sqrt{2 \pi} \int_{0}^{\infty} f(2 \pi u) d u$ and, by the Fourier inversion formula (2.6), $\frac{1}{2} \sqrt{2 \pi} f(0)=\int_{0}^{\infty} \widehat{f}(v) d v$.

This equation can be conveniently be written as

$$
\sqrt{2 \pi} \int_{0}^{\infty} f(2 \pi u) d \mu(u)=\int_{0}^{\infty} \widehat{f}(v) d \mu(v),
$$

Further, as $f \in \mathcal{S}$ implies $f^{\prime}, \widehat{f}^{\prime} \in L^{1}(\mathbb{R})$ and $\lim _{u \rightarrow \infty} f(u)=\lim _{v \rightarrow \infty} \widehat{f}(v)=0$, the two integrals can be converted into ordinary Lebesgue integrals by partial integration, namely,

$$
\begin{aligned}
\sqrt{2 \pi} \int_{0}^{\infty} f(2 \pi u) d \mu(u) & =\left.\sqrt{2 \pi} f(2 \pi u) \mu(u)\right|_{0} ^{\infty}-(2 \pi)^{3 / 2} \int_{0}^{\infty} \mu(u) f^{\prime}(2 \pi u) d u \\
& =-(2 \pi)^{3 / 2} \int_{0}^{\infty} \mu(u) f^{\prime}(2 \pi u) d u
\end{aligned}
$$

and similarly,

$$
\int_{0}^{\infty} \widehat{f}(v) d \mu(v)=-\int_{0}^{\infty} \mu(v) \widehat{f}^{\prime}(v) d v .
$$

Hence we obtain the following "integrated form" of PSF,

$$
(2 \pi)^{3 / 2} \int_{0}^{\infty} \mu(u) f^{\prime}(2 \pi u) d u=\int_{0}^{\infty} \mu(v) \widehat{f}^{\prime}(v) d v .
$$

For the Gauß-Weierstraß kernel $g_{\rho}$ of (2.12), (2.13) with $\rho=\pi t^{2}, t>0$, this identity reads,

$$
-2 \pi t^{-3} \int_{0}^{\infty} u \mu(u) e^{-\pi t^{-2} u^{2}} d u=-2 \pi t^{2} \int_{0}^{\infty} v \mu(v) e^{-\pi t^{2} v^{2}} d v .
$$


Now we multiply both sides of (4.6) by $-t^{-\sigma}, \sigma>0$, integrate with respect to $t$ from 0 to $\infty$, and interchange the order of integration, which is allowed by the FubiniTonelli theorem [40, (21.13)]. This yields for the left-hand side by a simple change of variables

$$
2 \pi \int_{0}^{\infty} u \mu(u) \int_{0}^{\infty} t^{-3-\sigma} e^{-\pi t^{-2} u^{2}} d t d u=\frac{1}{2} \pi^{-\sigma / 2} \Gamma\left(\frac{\sigma}{2}\right) \sigma \int_{0}^{\infty} u^{-\sigma-1} \mu(u) d u,
$$

and for the right-hand side

$$
\begin{aligned}
& 2 \pi \int_{0}^{\infty} v \mu(v) \int_{0}^{\infty} t^{2-\sigma} e^{-\pi t^{2} v^{2}} d t d v \\
& \quad=\frac{1}{2} \pi^{-\frac{1-\sigma}{2}} \Gamma\left(\frac{1-\sigma}{2}\right)(\sigma-1) \int_{0}^{\infty} v^{\sigma-2} \mu(v) d v .
\end{aligned}
$$

Using the representation (4.3) for the $\zeta$-function, the functional equation follows for $s=\sigma$ with $0<\sigma<1$, and analytic continuation yields the general result.

Proof of $F E R Z \Rightarrow P S F$ It is well-known that an infinite series can be transformed into an integral involving $\zeta(s)$ (as well as vice versa!) using Mellin transform methods; see e.g. [19]. Formally one proceeds as follows: To sum

$$
g(x):=\sum_{k=1}^{\infty} f(k x) \quad\left(x \in \mathbb{R}_{+}\right)
$$

one first applies the Mellin transform

$$
\mathcal{M}(f)(s) \equiv \widehat{f}_{M}(s):=\int_{0}^{\infty} f(u) u^{s-1} d u \quad(s=\sigma+i \tau \in \mathbb{C})
$$

on both sides to yield

$$
\widehat{g}_{M}(s)=\sum_{k=1}^{\infty} \frac{1}{k^{s}} \widehat{f}_{M}(s)=\widehat{f}_{M}(s) \zeta(s)
$$

since the Mellin transform of $f(k x)$, where $k>0$, is $k^{-s} \widehat{f}_{M}(s)$. The Mellin inversion formula

$$
f(x)=\frac{1}{2 \pi i} \int_{c-i \infty}^{c+i \infty} \widehat{f_{M}}(s) x^{-s} d s \quad(c>0)
$$

would then lead to

$$
g(x)=\sum_{k=1}^{\infty} f(k x)=\frac{1}{2 \pi i} \int_{c-i \infty}^{c+i \infty} \widehat{f}_{M}(s) \zeta(s) x^{-s} d s \quad(c>1) .
$$


In our situation, to work with the sum (4.8) we need to move the integration contour from $c+i t$ to $1-c+i t,-\infty<t<+\infty$. The equation for $x=2 \pi$ then becomes

$$
\sum_{k=1}^{\infty} f(2 k \pi)=\frac{1}{2 \pi i} \int_{1-c-i \infty}^{1-c+i \infty} \widehat{f_{M}}(s) \zeta(s)(2 \pi)^{-s} d s+S
$$

where $S$ accounts for the sum of the residues of the integrand due to the contour shift. The change of variable $s \mapsto 1-s$ in the integral causes the appearance of a term $\zeta(1-s)$ in the integrand. The functional equation is used at this point, to make the term $\zeta(s)$ reappear. But, as Eq. (4.8) shows, the inverse Mellin transform of a product involving $\zeta(s)$ represents a series $\sum h(k)$, for some function $h$. This function will turn out to be the Fourier transform of $f$, so that

$$
\sum_{k=1}^{\infty} f(2 k \pi)=\sum_{k=1}^{\infty} f^{\wedge}(k)+S
$$

A computation will show that the residues are $R_{0}=-\frac{1}{2} f(0)$ and $R_{1}=\frac{1}{2 \pi} \int_{0}^{\infty}$ $f(x) d x$, and PSF will then follow.

To carry out the above sketch of the proof and to justify these steps, consider a locally integrable function $f(x)$. Let it be $\mathcal{O}\left(x^{-a}\right)$ as $x \rightarrow 0$ and $\mathcal{O}\left(x^{-b}\right)$ as $x \rightarrow \infty$ for some $a<b$. The Mellin transform $\widehat{f}_{M}(s)$ then exists for any complex $s=\sigma+i \tau$ in the fundamental strip $a<\sigma<b$. This follows from

$$
\begin{aligned}
\left|\int_{0}^{\infty} f(u) u^{s-1} d u\right| & \leq \int_{0}^{1}|f(u)| u^{\sigma-1} d u+\int_{1}^{\infty}|f(u)| u^{\sigma-1} d u \\
& \leq A \int_{0}^{1} u^{-a+\sigma-1} d u+B \int_{1}^{\infty} u^{-b+\sigma-1} d u
\end{aligned}
$$

since the first integral exists if $\sigma>a$ and the second if $\sigma<b$. If, in addition, $f(y)$ is of bounded variation in a neighbourhood of $y=x$, the Mellin inversion formula holds, in the form

$$
\frac{f\left(x^{+}\right)+f\left(x^{-}\right)}{2}=\frac{1}{2 \pi i} \int_{\sigma-i \infty}^{\sigma+i \infty} \widehat{f}_{M}(s) x^{-s} d s,
$$

for $a<\sigma<b$. To justify it one may appeal to the relation between the Mellin and the Fourier transform, which is revealed by the change of variables $x=e^{-y}$, since, as we have seen, $f(x) x^{\sigma-1} \in L^{1}(0, \infty)$ under the stated hypothesis.

Now, if the intersection of the fundamental strip $a<\sigma<b$ with the half-plane $\sigma>1$ is nonempty, and if $s$ belongs to that intersection, then (4.7) holds because there exists under the stated conditions

$$
\sum_{k=1}^{\infty}\left|\frac{1}{k^{s}}\right| \int_{0}^{\infty}\left|u^{s-1} f(u)\right| d u .
$$


If $g$ is a continuous function of bounded variation in a neighbourhood of $x$, the application of the Mellin inversion formula to (4.7) then leads to the identity (4.8), that is,

$$
g(x)=\sum_{k=1}^{\infty} f(k x)=\frac{1}{2 \pi i} \int_{\sigma-i \infty}^{\sigma+i \infty} \widehat{f_{M}}(s) \zeta(s) x^{-s} d s .
$$

To continue with the proof, let $f$ be an even function in the Schwartz space $\mathcal{S}$. Then, the Mellin transform of $f$ exists for all $s=\sigma+i t$ with $\sigma>0$. Below we will have to integrate $\widehat{f}_{M}(s)$ in a region contained in the left semiplane, so we need to investigate its analytic extension there. The necessary information can be obtained through an integration by parts of the Mellin integral,

$$
\widehat{f_{M}}(s)=-\frac{1}{s} \int_{0}^{\infty} f^{\prime}(x) x^{s} d x=-\frac{1}{s} \widehat{f}_{M}^{\prime}(s+1) .
$$

The procedure can be repeated to give

$$
\widehat{f}_{M}(s)=\frac{(-1)^{r}}{s(s+1) \cdots(s+r-1)}{\widehat{\left[f^{(r)}\right]}}_{M}(s+r) \quad(r \in \mathbb{N}) \text {. }
$$

This provides the desired analytic extension and shows that $\widehat{f}_{M}(s)$ may have simple poles at the non-positive integers. The residue at $s=0$ is given by

$$
\lim _{s \rightarrow 0} s\left(-\frac{1}{s} \widehat{f}_{M}^{\prime}(s+1)\right)=-\widehat{f}_{M}^{\prime}(1)=-\int_{0}^{\infty} f^{\prime}(x) d x=f(0) .
$$

Although we will not need any other residues, one could easily check that the residue at $s=-n$ is $\frac{1}{n !} f^{(n)}(0)$.

We are now ready to return to the fundamental transformation (4.8) with $x=2 \pi$. We take an arbitrary $1<c<2$ and change the contour,

$$
\frac{1}{2 \pi i} \int_{c-i \infty}^{c+i \infty} \widehat{f_{M}}(s) \zeta(s)(2 \pi)^{-s} d s=\frac{1}{2 \pi i} \int_{1-c-i \infty}^{1-c+i \infty} \widehat{f}_{M}(s) \zeta(s)(2 \pi)^{-s} d s+S
$$

where $S$ is the sum of the residues of $\widehat{f}_{M}(s) \zeta(s)(2 \pi)^{-s}$ at the poles in the strip $1-c<\sigma<c$. There are two such poles: one at $s=1$, due to $\zeta(s)$, and another at $s=0$, due to $\widehat{f}_{M}(s)$. The residue of the integrand at the former is

$$
R_{1}=\frac{1}{2 \pi} \widehat{f}_{M}(1)=\frac{1}{2 \pi} \int_{0}^{\infty} f(x) d x
$$

and at the latter

$$
R_{0}=f(0) \zeta(0)=-\frac{1}{2} f(0)
$$


in view of (4.10). As a result, (4.8) becomes

$$
\sum_{k=1}^{\infty} f(2 k \pi)=\frac{1}{2 \pi i} \int_{1-c-i \infty}^{1-c+i \infty} \widehat{f_{M}}(s) \zeta(s)(2 \pi)^{-s} d s+\frac{1}{2 \pi} \int_{0}^{\infty} f(x) d x-\frac{1}{2} f(0) .
$$

We now replace $s \mapsto 1-s$ in the integral

$$
\begin{aligned}
& \sum_{k=1}^{\infty} f(2 k \pi) \\
& =\frac{1}{2 \pi} \int_{0}^{\infty} f(x) d x-\frac{1}{2} f(0)+\frac{1}{2 \pi i} \int_{c-i \infty}^{c+i \infty} \widehat{f}_{M}(1-s) \zeta(1-s)(2 \pi)^{s-1} d s
\end{aligned}
$$

and use the functional equation in the form (1.16) to deduce

$$
\begin{aligned}
& \sum_{k=1}^{\infty} f(2 k \pi) \\
& =\frac{1}{2 \pi} \int_{0}^{\infty} f(x) d x-\frac{1}{2} f(0)+\frac{1}{2 \pi i} \int_{c-i \infty}^{c+i \infty} \widehat{f}_{M}(1-s) 2 \cos \left(\frac{\pi s}{2}\right) \Gamma(s) \zeta(s) d s
\end{aligned}
$$

According to the principle expressed by (4.8), the last integral represents the sum of a series,

$$
\frac{1}{2 \pi i} \int_{c-i \infty}^{c+i \infty} \widehat{f_{M}}(1-s) 2 \cos \left(\frac{\pi s}{2}\right) \Gamma(s) \zeta(s) d s=\sum_{k=1}^{\infty} h(k) .
$$

The question is: what is the relation between $h$ and $f$ ? The elementary Mellin transform formula

$$
\int_{0}^{\infty} \cos (a x) x^{s-1} d x=a^{-s} \cos \left(\frac{\pi s}{2}\right) \Gamma(s) \quad(0<\Re s<1)
$$

shows that

$$
\begin{aligned}
\int_{0}^{\infty} \widehat{f}(v) v^{s-1} d v & =2 \int_{0}^{\infty}\left(\frac{1}{\sqrt{2 \pi}} \int_{0}^{\infty} f(t) \cos (v t) d t\right) v^{s-1} d v \\
& =\frac{2}{\sqrt{2 \pi}} \int_{0}^{\infty} f(t)\left(\int_{0}^{\infty} \cos (v t) v^{s-1} d v\right) d t \\
& =\frac{2}{\sqrt{2 \pi}} \cos \left(\frac{\pi s}{2}\right) \Gamma(s) \int_{0}^{\infty} f(t) t^{-s} d t \\
& =\frac{2}{\sqrt{2 \pi}} \cos \left(\frac{\pi s}{2}\right) \Gamma(s) \widehat{f}_{M}(1-s) .
\end{aligned}
$$


In order to justify the interchange of the integration order, first note that

$$
\begin{aligned}
I & :=\int_{0}^{\infty} f(t)\left(\int_{0}^{\infty} \cos (v t) v^{s-1} d v\right) d t \\
& =\int_{0}^{\infty} f(t) \lim _{R \rightarrow \infty}\left(\int_{0}^{R} \cos (v t) v^{s-1} d v\right) d t \\
& =\lim _{R \rightarrow \infty} \int_{0}^{\infty} f(t)\left(\int_{0}^{R} \cos (v t) v^{s-1} d v\right) d t .
\end{aligned}
$$

Here the interchange of the limit with the outer integral is justified by Lebesgue's dominated convergence theorem, since

$$
\begin{aligned}
\left|f(t) \int_{0}^{R} \cos (v t) v^{s-1} d v\right| & =\left|t^{-s} f(t) \int_{0}^{R t} \cos (u) u^{s-1} d u\right| \\
& \leq c(s) t^{-\sigma}|f(t)| \in L^{1}(0, \infty),
\end{aligned}
$$

where the constant $c(s)$ is an upper bound for the continuous and bounded function $x \mapsto\left|\int_{0}^{x} \cos (u) u^{s-1} d u\right|$ on $[0, \infty)$.

Furthermore, the order of integration in the last line of (4.16) can be interchanged by Fubini's theorem, and one obtains

$$
I=\lim _{R \rightarrow \infty} \int_{0}^{R}\left(\int_{0}^{\infty} f(t) \cos (v t) d t\right) v^{s-1} d v
$$

which proves the desired interchange of integrals in (4.15).

Hence we have shown that

$$
\int_{0}^{\infty} \widehat{f}(v) v^{s-1} d v=\frac{2}{\sqrt{2 \pi}} \cos \left(\frac{\pi s}{2}\right) \Gamma(s) \widehat{f}_{M}(1-s) .
$$

Since we have used the elementary formula (4.14) to deduce this equation, it holds at first for $0<\Re s<1$. On the other hand, both sides of (4.17) define analytic functions for $\Re z>0$, and hence (4.17) is even valid for $\Re z>0$.

Now, equation (4.17) reveals that the factor that multiplies $\zeta(s)$ in the integrand of (4.13) is the Mellin transform of the Fourier transform $\widehat{f}$ of $f$. Thus (4.13) becomes

$$
\frac{1}{2 \pi i} \int_{c-i \infty}^{c+i \infty} \widehat{f}_{M}(1-s) 2 \cos \left(\frac{\pi s}{2}\right) \Gamma(s) \zeta(s) d s=\frac{1}{\sqrt{2 \pi}} \sum_{k=1}^{\infty} \widehat{f}(k) .
$$

Replacing it in (4.11) finally yields

$$
\sum_{k=1}^{\infty} f(2 k \pi)=\frac{1}{2 \pi} \int_{0}^{\infty} f(x) d x-\frac{1}{2} f(0)+\frac{1}{\sqrt{2 \pi}} \sum_{k=1}^{\infty} \widehat{f}(k) .
$$


Noting that $\frac{1}{2 \pi} \int_{0}^{\infty} f(x) d x=\frac{1}{2} \frac{1}{\sqrt{2 \pi}} \widehat{f}(0)$, the PSF (1.10) for even functions in the Schwarz space $\mathcal{S}$ and $x=0$ follows.

To extend this to arbitrary functions $f \in \mathcal{S}$, one decomposes $f$ in the form $f(t)=$ $\frac{1}{2}[f(t)+f(-t)]+\frac{1}{2}[f(t)-f(-t)]$, i. e., in an even and an odd part. Since both sums in (1.10) vanish for odd functions and $x=0$, PSF for $f \in \mathcal{S}$ and $x=0$ follows. To obtain PSF for arbitrary $x \in \mathbb{R}$, one replaces $f(\cdot)$ by $2 \pi f(x+2 \pi \cdot)$ as in the proof of $\mathrm{ASF} \Rightarrow \mathrm{PSF}$. Finally, one uses the fact that $\mathcal{S}$ is dense in $L^{1}(\mathbb{R})$ to deduce the general PSF for $f \in L^{1}(\mathbb{R})$; cf. Sect. 2.4.

Let us remark that a proof of PSF yields FERZ is to be found in Mordell [57], and the converse in Ferrar [30]. In his treatise Titchmarsh [82] presented seven different methods of proof of the functional equation; see also Kahane and Mandelbrojt [50], Bellman [8], Ivić [49], Patterson [66, Chapter 2], Karatsuba and Voronin [51], Rooney [75], Flajolet et al. [31], Brüdern [13, pp. 58-66], Newman [63]. Latter proofs are Knopp and Robins [55], Schuster [78], Murty [58] and Higgins [45].

Whereas several of the above mentioned books or papers contain a proof of $\mathrm{PSF} \Rightarrow \mathrm{FERZ}$, proofs of the converse $\mathrm{FERZ} \Rightarrow \mathrm{PSF}$, an important part of our procedure, are rarer. In fact, there are other proofs of FERZ $\Leftrightarrow$ PSF, but ours are fully detailed and more than just polished versions of others. Especially the delicate interchanges of the orders of summation and/or integration needed in the proofs are justified in great detail.

In his exhaustive report [44] on four papers of Hamburger [33-36] of 1921-1922 in connection with Riemann's functional equation, R. Higgins gave a complete review of Hamburger's collection of five equivalent results, namely that Riemann's functional equation for the zeta function, Jacobi's transformation formula for the elliptic theta function, the partial fractions expansion for the cotangent function, Poisson's summation formula and a special Fourier series are all equivalent. He also discussed these in the light of later contributions.

Here the basic earlier work being the "Lehrbuch der Thetafunktionen" by Krazer [56], papers by Siegel [79], those of Mordell [57] and Ferrar [30], as well as more results in the light of work by Doetsch [27] and Klusch [52,53]. Klusch proves many implications, and collects others, between well-known results in signal analysis, number theory and applied mathematics. In particular, he gives a direct proof of FERZ implies ASF, an open question at the time. These results do not seem to be at all well known.

\section{A proof of ASF}

In the previous section we have shown that the formulae of Sects. 1 or 3 can all be deduced from each other. Hence it suffices to prove one of them in order to verify all. We will now prove ASF under the weaker hypothesis that $f \in F^{2}$ and with a weaker conclusion on the convergence of the series. From that result, the statement (1.6) for $f \in F^{2} \cap S_{w}^{1}$ given in Sect. 1 is easily deduced. 
Theorem 1 For $w>0$ and $f \in F^{2}$, we have

$$
f(t)=\sum_{k \in \mathbb{Z}} f\left(\frac{k}{w}\right) \operatorname{sinc}(w t-k)+R_{w} f(t),
$$

where the series converges pointwise as the symmetric sum.

Proof It suffices to consider the case $w=1$. Employing the Fourier inversion theorem for $f \in F^{2}$, see [20, p. 214, Proposition 5.2.16], we may rewrite the remainder (1.2) as

$$
\begin{aligned}
R_{1} f(t) & =\frac{1}{\sqrt{2 \pi}}\left[\int_{\mathbb{R}} \widehat{f}(v) \mathrm{e}^{\mathrm{i} v t} \mathrm{~d} t-\sum_{k \in \mathbb{Z}} \int_{(2 k-1) \pi}^{(2 k+1) \pi} \widehat{f}(v) e^{\mathrm{i} t(v-2 \pi k)} \mathrm{d} v\right] \\
& =f(t)-\frac{1}{\sqrt{2 \pi}} \int_{\mathbb{R}} \widehat{f}(v) g(t, v) \mathrm{d} v
\end{aligned}
$$

where $g(t, \cdot)$ is the function obtained by restricting $\mathrm{e}^{\mathrm{i} t \cdot}$ to the interval $[-\pi, \pi)$ and extending it to $\mathbb{R}$ by $2 \pi$ periodic continuation. By a simple calculation we obtain the Fourier expansion

$$
g(t, v)=\sum_{k \in \mathbb{Z}} \operatorname{sinc}(t-k) \mathrm{e}^{i k v}
$$

Since $g(t, \cdot)$ is of bounded variation, the Fourier series converges and (5.2) holds at each point of continuity. Moreover, the partial sums

$$
g_{N}(t, v):=\sum_{|k| \leq N} \operatorname{sinc}(t-k) \mathrm{e}^{\mathrm{i} k v}
$$

are uniformly bounded, that is

$$
\left|g_{N}(t, v)\right| \leq C \quad(v \in \mathbb{R}, N \in \mathbb{N})
$$

for some constant $C$; see [85, p. 90, Thm. 3.7]. Since $\widehat{f} \in L^{1}(\mathbb{R})$, we see that $\widehat{f}(\cdot) g_{N}(t, \cdot)$ has an absolutely integrable majorant for all $N \in \mathbb{N}$. Therefore, Lebesgue's dominated convergence theorem allows us to conclude that

$$
\begin{aligned}
\frac{1}{\sqrt{2 \pi}} \int_{\mathbb{R}} \widehat{f}(v) g(t, v) \mathrm{d} v & =\lim _{N \rightarrow \infty} \frac{1}{\sqrt{2 \pi}} \int_{\mathbb{R}} \widehat{f}(v) g_{N}(t, v) \div \\
& =\lim _{N \rightarrow \infty} \sum_{|k| \leq N}\left\{\frac{1}{\sqrt{2 \pi}} \int_{\mathbb{R}} \widehat{f}(v) \mathrm{e}^{\mathrm{i} k v} \mathrm{~d} v\right\} \operatorname{sinc}(t-k) \\
& =\lim _{N \rightarrow \infty} \sum_{|k| \leq N} f(k) \operatorname{sinc}(t-k),
\end{aligned}
$$


where again the Fourier inversion theorem (2.6) has been used in the last step. Now the proof is completed by substituting the last expression in (5.1).

Let us mention that in the meantime some of the authors have shown that ASF is equivalent to the classical sampling theorem (1.7); see [14]. The above proof of ASF is, on the other hand, a Fourier analytic proof, fully independent of CSF and all the theorems of this paper.

\section{Epilogue: some possible generalizations}

The natural question arises as to whether the results above can be extended to higher dimensions or other settings. There are (at least) two ways : via functional analysis, based on r.k. theory, and via abstract harmonic analysis, using locally compact abelian (LCA) groups.

In the first of these generalizations, sampling itself is placed in the more general context of reproducing kernel Hilbert spaces of functions defined on an abstract set. In the second, the real line $\mathbb{R}$, which can be thought of as time domain, is replaced by an abstract LCA topological group $G$, as usual assumed to be Hausdorff. Functions are now defined on a LCA group, itself a fruitful generalization of the real line.

\subsection{Reproducing kernel theory}

The purpose of this section is to give some very brief and necessarily quite simplified background to Hilbert spaces with reproducing kernel. The material in this section, proofs etc., can be found in [42,77]. There are several studies in the literature of sampling to be found in the general area of reproducing kernel spaces, and we can ask whether results of the present paper might be given more general forms in that theory. For example, Nashed and his associates have given sampling theorems in certain Hilbert, Banach, Sobolev and translation invariant spaces, and addressed such probems as the construction of reproducing kernel Hilbert and Banach spaces with a given sampling set (see, e.g., [59]). Much of this work is quite technical and uses, e.g., iterative methods. Saitoh's theory of linear transformations of Hilbert space offers another possibility for this kind of extension (see, e.g., [77]).

\subsubsection{Hilbert spaces with reproducing kernel}

Let $K$ be a separable Hilbert space with inner product denoted by $\langle\cdot, \cdot\rangle_{K}$ and norm denoted by $\|\cdot\|_{K}$.

Definition 1 Let $K$ consist of complex valued functions defined on $E$, an abstract set. Then $K$ is said to have reproducing kernel if there exists a function $k: E \times E \mapsto \mathbb{C}$, such that $k(\cdot, t) \in K$ for every $t \in E$, and the reproducing equation

$$
f(t)=\langle f, k(\cdot, t)\rangle_{K}
$$


holds for every $f \in \mathrm{K}$. Such a space is called a reproducing kernel Hilbert space $(R K H S)$ and $k(s, t)$ is its reproducing kernel.

\subsubsection{A summary of the relevant Saitoh theory}

We now quote that part of Saitoh's fundamental theory of linear transformations of Hilbert space which provides a suitable setting for the present discussion ([77, Ch. 2]), but first some background is needed.

Let $\mathfrak{H}$ be a separable Hilbert space with inner product denoted by $\langle\cdot, \cdot\rangle_{\mathfrak{H}}$, and for each $t$ belonging to an abstract set $E$, let $\kappa_{t}$ be a mapping of $E$ into $\mathfrak{H}$. Then $k(s, t):=\left\langle\kappa_{t}, \kappa_{s}\right\rangle_{\mathfrak{H}}$ is defined on $E \times E$ and is called the kernel function of the map $\kappa_{t}$. This kernel function is a positive matrix [77, Ch. 2, Sect. 2] and as such it determines one and only one Hilbert space for which it is the reproducing kernel. This Hilbert space is denoted by $R_{k}$; it turns out to be the set of images of $\mathfrak{H}$ under the transformation

$$
(\mathcal{L} \varphi)(t):=\left\langle\varphi, \kappa_{t}\right\rangle_{\mathfrak{H}}, \quad(\varphi \in \mathfrak{H})
$$

and has reproducing kernel $k(s, t)=\left(\mathcal{L} \kappa_{t}\right)(s)$. This situation is governed by:

Theorem 2 (Saitoh) With the notations established above, $R_{k}$ is a Hilbert space which has the reproducing kernel $k(\cdot, \cdot)$, and is uniquely determined by this kernel. We have, for $f \in R_{k}$,

$$
\|f\|_{R_{k}}=\|\mathcal{L} \omega\|_{R_{k}} \leq\|\omega\|_{\mathfrak{H}},
$$

and there exists a unique member, $\omega_{0}$ say, of the class of all $\omega$ 's satisfying (6.2) such that

$$
f(t)=\left\langle\omega_{0}, \kappa_{t}\right\rangle_{\mathfrak{H}}, \quad(t \in E),
$$

and

$$
\|f\|_{R_{k}}=\left\|\omega_{0}\right\|_{\mathfrak{H}} .
$$

The reproducing equation for $R_{k}$ is

$$
f(t)=\langle f, k(\cdot, t)\rangle .
$$

It is often supposed that $\left\{\kappa_{t}\right\},(t \in E)$ is complete in $\mathfrak{H}$, a rather mild restriction. This means that the only possible $\omega$ in (6.2) is $\omega_{0}$, because from (6.1) the null space of $\mathcal{L}$ is $\{\theta\}$. Then $\mathcal{L}: \mathfrak{H} \mapsto R_{k}$ is an isometry and therefore bounded. It is clearly linear, one-to-one and 'onto'. Hence by the bounded inverse theorem, $\mathcal{L}^{-1}$ is bounded. These properties show that $\mathcal{L}$ is an isometric isomorphism of $\mathfrak{H}$ onto $R_{k}$.

\subsubsection{Possibilities for extension}

The r.k. theory outlined above is in some ways more general and in some ways less general than the LCA group setting. It is tied to Hilbert space methods and no extension beyond these methods is known to the authors. 
However, in order to find some process of extension beyond Hilbert space there might be a way forward if a regime such as the following could be established. To describe such a regime we will adopt the following notations.

Suppose that $\mathfrak{X}$ is a linear space, and let $\mathfrak{X}^{\text {ex }}$ denote a linear space that extends $\mathfrak{X}$; that is, $\mathfrak{X}$ is a subspace of $\mathfrak{X}^{\text {ex }}$. Again, suppose that $\mathcal{P}$ denotes a proposition associated with $\mathfrak{X}$; it might be, for example, the assertion that a certain formula holds for all members of $\mathfrak{X}$. Let $\mathcal{P}^{\text {ex }}$ denote an extension of $\mathcal{P}$, that is, a proposition associated with $\mathfrak{X}^{\mathrm{ex}}$ so chosen that it reduces to $\mathcal{P}$ for members of $\mathfrak{X}$.

With these notations, the following extension procedure can be suggested for the r.k. theory. Let $\mathfrak{H}^{\text {ex }}$ denote a linear space which extends $\mathfrak{H}$ in such a way as to extend the domain of $\mathcal{L}$, thus, $\mathcal{L}$ on this larger domain induces an extension $R_{k}^{\text {ex }}$ of $R_{k}$. Let $\mathcal{P}$ denote a proposition associated with $R_{k}$, and $\mathcal{P}^{\text {ex }}$ a proposition associated with $R_{k}^{\text {ex }}$, asserting that $\mathcal{P}$ holds for $R_{k}^{\mathrm{ex}}$ but only in an approximate form when associated with $R_{k}^{\mathrm{ex}} \backslash R_{k}$.

A scheme of this kind might afford some way of generalizing results found in the main body of the paper, such as the passage from the classical to the approximate sampling theorem $(1.7) /(1.6)$, or the reproducing equation (1.8) to the approximate reproducing equation (1.9). But the construction of such a scheme is an open problem, no doubt a rather difficult one.

\subsubsection{A dictionary}

The question arises as to whether the ASF of (1.6) and ARKF of (1.9), introduced in this paper, as well as GPDF of (1.1) can be built into the more recent reproducing kernel Banach space theory (see e.g. $[1,32,37,47,60,61]$ ), since the Hilbert space approach may not suffice. Its non-constructed but practical applications would be precisely the present six theorems under discussion. Perhaps an extension of the Banach space theory may also be necessary. It is seemingly a wide open field (Table 1).

\subsection{Abstract harmonic analysis}

In abstract harmonic analysis, the real line in classical Fourier analysis is replaced by an LCA group. A very brief description is now given, following Rudin's notation and terminology in [76]. Further details can also be found in $[4,25,38,39,73]$ and briefer

Table 1 A classical-reproducing kernel theory dictionary

\begin{tabular}{ll}
\hline Classical & r.k. theory \\
\hline $\mathbb{R}$ & $E$, an abstract set \\
$L^{2}(-\pi, \pi)$ & $\mathfrak{H}$, a separable Hilbert space \\
$(2 \pi)^{-1 / 2} e^{-i t \cdot} \chi_{[-\pi, \pi]}(\cdot)$ & $\kappa_{t} \in \mathfrak{H},(t \in E)$ \\
reproducing kernel: $\operatorname{sinc}(s-t)$ & $k(s, t)=\left\langle\kappa_{t}, \kappa_{s}\right\rangle_{\mathfrak{H}}$ \\
$\mathcal{F}^{-1}$, inverse Fourier transform & $\mathcal{L}$ \\
$\hat{B}_{\sigma}^{p}$ & $R_{k}$ \\
reproducing equation $f(t)=\langle f, \operatorname{sinc}(\cdot-t)\rangle$ & $f(t)=\langle f, k(\cdot, t)\rangle_{R_{k}}$ \\
\hline
\end{tabular}


accounts are in $[6,7,28]$. The website [65] of the Numerical Harmonic Analysis Group (NuHAG) at the University of Vienna is a comprehensive and up-to-date source of information about all aspects of sampling theory.

\subsubsection{Locally compact abelian groups}

The property of being an LCA group is often preserved under processes that are subject to natural technical restrictions. Thus closed subgroups of an LCA group are also LCA, as are quotient groups of closed subgroups and so on. LCA groups enjoy a translation invariant measure $m_{G}$, called Haar measure, which is unique up to a multiplicative constant (for the real line, the Haar measure is the familiar Lebesgue measure). As with Lebesgue measure, Haar measure gives rise to an integral $\int_{G} f(x) d m_{G}(x)=\int_{G} f$; the notation $f \in L^{p}(G)$ is used if $\int_{G}|f|^{p}<\infty, p \geq 1$. As usual the statement that a property holds for (Haar) almost all points in a subset of $G$ means that it holds for all points in the set except for a set of (Haar) measure 0 .

\subsubsection{The abstract Fourier transform}

The exponential function $t \mapsto e^{i u t}$ is replaced by a continuous homomorphism or character $\gamma: G \rightarrow \mathbb{S}^{1}$, which takes values on the unit circle. Under pointwise multiplication, these homomorphisms form an abelian group $G^{\wedge}:=\Gamma$ which is locally compact with the compact-open topology. Greek letters such as $\gamma$ and $\lambda$ will be used for elements in $\Gamma$, which is called the dual group of $G$ and corresponds to the frequency domain. The dual of $\Gamma$ is isomorphic to $G$. This duality allows the value $\gamma(x), x \in G$, of a character $\gamma$ to be written as an ordered pair $(x, \gamma)$, where $(x, \gamma)\left(x^{\prime}, \gamma\right)=\left(x+x^{\prime}, \gamma\right)$ and similarly for $\gamma$.

The Fourier transform $\widehat{f}: \Gamma \rightarrow \mathbb{C}$ of the function $f \in L^{1}(G)$, defined by

$$
\widehat{f}(\gamma)=\int_{G} f(x)(x,-\gamma) d m_{G}(x),
$$

is continuous and vanishes at $\infty$. For each $\psi$ in $L^{1}(\Gamma)$, the inverse Fourier transformfunction $\psi^{\vee}: G \rightarrow \mathbb{C}$ is defined by

$$
\psi^{\vee}(x)=\int_{\Gamma} \psi(\gamma)(x, \gamma) d m_{\Gamma}(\gamma) .
$$

The Haar measure $m_{\Gamma}$ on $\Gamma$ can be normalized so that the inversion formula

$$
f(x)=\int_{\Gamma} \widehat{f}(\gamma)(x, \gamma) d m_{\Gamma}(\gamma)
$$

holds almost always for suitable $f=\psi^{\vee}$ (see [76, Sect. 1.5] or [39, Thm. 31.17]).

These ideas are the basis of a beautiful and broad abstract analogue of classical Fourier analysis that retains the principal results of classical theory. Thus the abstract 
Table 2 Some LCA groups, their duals, measures and characters

\begin{tabular}{lllll}
\hline Group $G$ & measure $m_{G}$ & dual $\Gamma$ & measure $m_{\Gamma}$ & character $(x, \gamma)$ \\
\hline $\mathbb{R}$ & Lebesgue & $\mathbb{R}$ & Lebesgue & $e^{i v \xi}$ \\
$\mathbb{R}^{r}$ & Lebesgue & $\mathbb{R}^{r}$ & Lebesgue & $e^{i \mathbf{v} \cdot \boldsymbol{\xi}}$ \\
$\mathbb{Z}^{s}$ & Point measure & $\mathbb{T}^{s}$ & induced Lebesgue & $e^{i \mathbf{k} \cdot \boldsymbol{\xi}}$ \\
$\mathbb{Z}_{n}^{s}$ & Point measure & $\mathbb{Z}_{n}^{s}$ & point measure & $e^{i \mathbf{k} \cdot \boldsymbol{v}}$ \\
\hline
\end{tabular}

Table 3 A classical-abstract dictionary for sampling theory

\begin{tabular}{ll}
\hline Classical & Abstract \\
\hline Time domain $\mathbb{R}$ & LCA group $G$ \\
Frequency domain $\mathbb{R}$ & dual LCA group $G^{\wedge}=\Gamma$ \\
Lebesgue measure $|\cdot|$ & Haar measure $m_{G}$ \\
$e^{i x u}$ & character $(x, \gamma)$ \\
$\widehat{f}(u):=\int_{\mathbb{R}} f(x) e^{-i x u} d x$ & $\widehat{f}(\gamma):=\int_{G} f(x)(x,-\gamma) d m_{G}(x)$ \\
$2 w \mathbb{Z}$ discrete subgroup of $\mathbb{R}$ & $\Lambda$ discrete subgroup (lattice) of $\Gamma$ \\
$\mathbb{R} /(2 w \mathbb{Z}) \cong \mathbb{S}^{1}$ & $\Gamma / \Lambda$ compact abelian group \\
$(-\pi w, \pi w]$ transversal of $\mathbb{R} /(2 \pi w \mathbb{Z})$ & $\Omega$ transversal of $\Gamma / \Lambda$ \\
$\mathbb{Z} / w=(2 w \pi \mathbb{Z})^{\perp}$ sampling set & $H=\Lambda^{\perp}=\{h \in G:(h, \lambda)=1, \lambda \in \Lambda\}$ \\
$|(-\pi w, \pi w]|=2 \pi w$ & $m_{\Gamma}(\Omega)<\infty$ \\
$\sum_{k \in \mathbb{Z}} g(k / 2 w)$ & $\int_{H} g(h) d m_{H}(h)=m_{H}(\{0\}) \sum_{h \in H} g(h)$ \\
$\widehat{B}_{\pi}^{p}$ band-limited signals & $\widehat{B}^{p}(G)$ \\
$F^{p}$ includes non band-limited signals & $F^{p}(G)$ \\
$\ell^{p}(\mathbb{Z} / w)$ & $\ell^{p}(H)$ \\
\hline
\end{tabular}

Fourier-Plancherel transform can be defined on $L^{2}(G)$ and analogues of the classical Fourier-Plancherel theorem $\left(\int_{G}|f|^{2}=\int_{\Gamma}\left|f^{\wedge}\right|^{2}\right)$ [39, Sect. 31], [76] and the Parseval theorem hold (here and where appropriate ${ }^{-}$is the Fourier-Plancherel transform). The abstract Poisson summation formula also holds under certain integrability conditions [76].

The general and unifying framework offered by abstract Fourier analysis includes $n$-dimensional Euclidean space as an important special case. Some other concrete examples of LCA groups, their duals, measures and characters are provided in Table 2; $\mathbb{Z}_{n}$ is the finite additive group of residues $\bmod n$ and $\mathbb{T}^{r}=\mathbb{R}^{r} / \mathbb{Z}^{r}$ is the $r$-dimensional torus which we will take to be $[-1 / 2,1 / 2]^{r}$, the $r$-fold product of the unit interval with endpoints identified. In particular, $\mathbb{T}^{1} \cong \mathbb{S}^{1}$, the unit circle. This normalization differs slightly from that adopted in Table 3.

\subsection{Abstract sampling theory}

Sampling theory fits naturally into the abstract Fourier analysis setting, as the table above indicates, with different LCA groups giving rise to a variety of seemingly 
disparate sampling results [4]. Of course, there are some limitations to be expected in such a general theory. To explain it more fully, some additional definitions and notation are needed.

Let $\Lambda$ be a lattice in $\Gamma$, i.e., a countable discrete subgroup of $\Gamma$ with compact quotient $\Gamma / \Lambda$. Note that it is appropriate to work with the dual group $\Gamma$ here, as it corresponds to the frequency domain. The lattice $\Lambda$ corresponds to the sampling set in time domain.

The annihilator

$$
\Lambda^{\perp}:=H=\{h \in G:(h, \lambda)=1 \text { for all } \lambda \in \Lambda\}
$$

of $\Lambda$ is a closed subgroup which we will write $H$ and which satisfies $H^{\perp}=\Lambda^{\perp \perp}=$ $\Lambda$ [76, Lemma 2.1.3]. The annihilator $H$ of $\Lambda$ is isomorphic to the dual of $\Gamma / \Lambda$, i.e., $H \cong(\Gamma / \Lambda)^{\wedge}$ (algebraically and topologically) [76, Thm. 2.1.2] and is identified with the dual $(\Gamma / \Lambda)^{\wedge}$. The annihilator $H$ plays the role of the sampling set in the case of the LCA group $G$. When $G=\Gamma=\mathbb{R}$ with lattice $\Lambda=2 \pi w \mathbb{Z}, H$ reduces to the sampling set $\mathbb{Z} / w$ in (1.7).

The Haar measure $m_{\Lambda}$ on $\Lambda$ will be normalized so that the Weil coset decomposition formula

$$
\int_{\Gamma} \varphi(\gamma) d m_{\Gamma}(\gamma)=\int_{\Gamma / \Lambda} \int_{\Lambda} \varphi(\gamma+\lambda) d m_{\Lambda}(\lambda) d m_{\Gamma / \Lambda}([\gamma])
$$

holds for suitable $\varphi: \Gamma \rightarrow \mathbb{C}$ (see [39, Sect. 28.54 (iii)] or [73, Sect. 2.7.3]). The Haar measure $m_{\Gamma / \Lambda}$ of $\Gamma / \Lambda$ is normalized so that the corresponding inversion formula (6.6) holds (with $G$ replaced by $\Gamma / \Lambda$ and $\Gamma$ by $H$ ). The Haar measures of $H$ and $G / H$ are also normalized so that the Weil formula (6.7) holds for $G$ and $H$ and so that the corresponding inversion formula (6.6) holds (with $G$ replaced by $G / H$ and $\Gamma$ by $\Lambda$ ).

The quotient group $\Gamma / \Lambda$ has a complete set of coset representatives, also referred to as a transversal, the terminology we will use. Transversals are not unique and there is always a measurable one [29] which we will choose. Note that while the quotient group is compact, the transversal is only of finite measure; indeed unbounded transversals are possible (see [5, Sect. 4]). By definition, a transversal $\Omega$ consists of just one point from each distinct coset $[\gamma]=\Lambda+\gamma$, i. e., $\Omega \cap(\Lambda+\gamma)$ consists of a single point in $\Omega$. Thus translates of $\Omega$ by non-zero elements in $\Lambda$ are disjoint from $\Omega$. Using (6.7), it is straightforward to verify that the Haar measure of the transversal $\Omega$ of $\Gamma / \Lambda$ satisfies $m_{\Gamma}(\Omega)=m_{\Lambda}(\{0\}) m_{\Gamma / \Lambda}(\Gamma / \Lambda)=m_{\Lambda}(\{0\}) / m_{H}(\{0\})<\infty$.

When the group $G$ is discrete and countable, the integral over $G$ reduces to a sum. This is particularly exploited for the subgroup $\Lambda$ of $\Gamma$ which is discrete and assumed countable, so that the integral

$$
\int_{\Lambda} \varphi(\lambda) d m_{\Lambda}(\lambda)=m_{\Lambda}(\{0\}) \sum_{\lambda \in \Lambda} \varphi(\lambda)
$$

where $m_{\Lambda}(\{0\})$ is the (discrete) measure of $\{0\}$. 


\subsubsection{Exact sampling in abstract harmonic analysis}

Kluvánek [54] was the first to place sampling theory in the LCA group setting by establishing the abstract analogue of the classical sampling theorem (i.e., the exact sampling formula (1.7)). He considered LCA groups $G$, each with dual $\Gamma$ having a lattice $\Lambda$ (so that $\Lambda$ is a discrete, countable subgroup of $\Gamma$ and $\Gamma / \Lambda$ is compact). A measurable transversal $\Omega$, with necessarily finite Haar measure $m_{\Gamma}(\Omega)$, is taken to represent the spectrum of $f$. Then Kluvánek took as an analogue of the class $\widehat{B}_{\pi w}^{2}$ (defined in Sect. 2.1 and called $P W_{w}$ elsewhere) of band-limited functions, the class

$$
\widehat{B}_{\Omega}^{2}(G):=\left\{f \in L^{2}(G) \cap C(G): \operatorname{supp} \widehat{f} \subseteq \Omega\right\}
$$

Kluvánek showed that functions $f$ in $\widehat{B}^{2}(G)$ have the representation $f=S_{H} f$, where $S_{H} f$ is the abstract analogue of the classical sampling series $S_{w} f(2.18)$ and is given by

$$
\left(S_{H} f\right)(x):=\frac{1}{m_{\Gamma}(\Omega)} \sum_{h \in H} f(h) \chi_{\Omega}(x-k),
$$

where convergence is in the $L^{2}$ sense and uniform. He further showed that $\|f\|_{G}^{2}=$ $\int_{G}|f|^{2}=\sum_{h \in H}|f(h)|^{2}$, the abstract sampling analogue for Plancherel's theorem, equivalent to that for Parseval's theorem: $\int_{G} f \bar{g}=\sum_{h \in H} f(h) \bar{g}(h)$ and obviating the need for the $\ell^{2}(H)$ summability condition.

The discrete Fourier transform, involving essentially $\mathbb{Z}$ and $\mathbb{S}^{1}$, also fits naturally into this framework. The abstract analogue would involve a discrete abelian group and a compact group respectively. A similar pattern holds for multi-dimensional signals in higher dimensions.

\subsubsection{Approximate sampling in abstract harmonic analysis}

More recently, abstract analogues of the approximate or generalized sampling formula (ASF) have been established. Faridani [28] proved a very general approximation theorem under an integrability condition. In [7,26], an abstract approximate sampling theorem has been established under the square summability condition $f \in \ell^{2}(H)$ (in Sect. 2.1, $f \in S_{w}^{2}=\ell^{2}(\mathbb{Z} / w)$ ). The generalization of the classical sets $F^{p}$ and $S_{w}^{p}, p=1,2$ (defined in Sect. 2.1), which are appropriate for extending approximate sampling theory to LCA groups, are

$$
F^{p}(G):=\left\{f \in L^{p}(G) \cap C(G): \widehat{f} \in L^{1}(\Gamma)\right\}
$$

and

$$
S_{H}^{p}(G):=\left\{f: G \rightarrow \mathbb{C}: f \in \ell^{p}(H)\right\}=\ell^{p}(H) .
$$

The abstract analogue of the ASF (1.6), is now given. 
Theorem 3 Suppose that the dual $\Gamma$ of the locally compact abelian group $G$ has a discrete subgroup $\Lambda$ with $\Gamma / \Lambda$ compact and that $\Omega$ is a measurable transversal of $\Gamma / \Lambda$. Then each $f \in F^{2}(G) \cap \ell^{2}(H)$ has a representation

$$
f=S_{H} f+R_{H} f
$$

where $H=\Lambda^{\perp}$ and $\left|\left(R_{H} f\right)(x)\right| \leq 2 \int_{\Gamma \backslash \Omega}|\widehat{f}|$.

An asymptotic formula needs more information [7, Thms. 5, 6].

A norm result for $f$ corresponding to Kluvánek's theorem is not known, although putting $f=g$ in the generalized or approximate Parseval formula (1.1) suggests that modulo some summability conditions

$$
\int_{G}|f|^{2}=\sum_{h \in H}|f(h)|^{2}-E_{1}+E_{2}
$$

where

$$
E_{1}=\frac{1}{m_{\Gamma}(\Omega)} \sum_{h \in H} f(h) \int_{\Gamma \backslash \Omega}(\bar{f})^{\wedge}(\gamma)(h, \gamma) d \gamma, E_{2}=\int_{G}\left(R_{H} f\right) \bar{f}
$$

This would imply an abstract Parseval type result and merits investigation. However, the proof in [18] of the formula (1.1) uses some subtle Fourier analysis similar to that employed by Brown [11,12] and Boas [9] so that extra hypotheses would be very likely be needed.

Turning to the question of extending the equivalence results in Sect. 3, it could be conjectured possible that under suitable hypotheses, the abstract analogue of ASF (1.6) is equivalent to those of ARKF (1.9) and GPDF (1.1). However, the proof in [45] that the functional equation of the zeta function $\zeta$ is equivalent to the classical exact sampling theorem is far from direct, requiring long chains of implications and many results from complex analysis. Thus proving an LCA group result would appear to be very difficult, undoubtedly involving extra hypotheses. Moreover, even defining a suitable zeta function for LCA groups seems problematic as the natural numbers $\mathbb{N}$ form a multiplicative semigroup generated by primes. Nevertheless, the classical situation suggests some interesting lines of inquiry and is a useful guide to the further understanding of abstract Fourier analysis.

\subsection{Sampling in Hilbert spaces, on Riemannian manifolds and graphs}

Let $L$ be a self-adjoint operator in a Hilbert space $H$ and $P_{\lambda}, \lambda \in \mathbb{R}$, its spectral resolution of identity. With every vector $f$ in $H$ the family of projectors $P_{\lambda}$ associates a measure on $\mathbb{R}$ which is given by the formula $\left\langle P_{\lambda} f, f\right\rangle$, where $\langle\cdot, \cdot\rangle$ is the inner product in $H$. Using this framework I. Pesenson introduced Paley-Wiener-type subspaces $P W_{\omega}(L), \quad \omega>0$, by saying that $f$ belongs to $P W_{\omega}(L)$ if and only if the measure $\left\langle P_{\lambda} f, f\right\rangle$ has support in $[-\omega, \omega]$. It turned out that these subspaces enjoy all the basic properties of the classical Paley-Wiener spaces and in particular they are suitable for 
development of a rich sampling and approximation theories. On the level of abstract Hilbert spaces it was done by Pesenson in $[67,70]$. Moreover, he specified this abstract setup in a number of very important situations such as: Riemannian compact and noncompact manifolds of bounded geometry [69], stratified Lie groups [68], combinatorial and quantum graphs [71,72].

\section{A short biography of Wolfgang Splettstößer}

Wolfgang Splettstößer, born in Düsseldorf on June 19, 1950, received his Abitur from the Leibniz Gymnasium in Düsseldorf in 1968, began studying mathematics at the RWTH Aachen in 1970, and received the Dipl. Math. degree with the bestowal of the "Springorum Denkmünze" in 1975. That year the electrical engineer Otto Lange (Aachen/TU Hamburg-Harburg) suggested one ought to examine in detail the broad area of the Shannon sampling theorem. Having attended the "1. Aachener Kolloquium", devoted to "Special problems of signal theory", conducted by the late Hans Dieter Lüke that March, the senior author (PLB) realized that the topic was indeed an important one and Wolfgang Splettstößer would be just the right person for this field. Within a short time he performed pioneering research in sampling theory which led to his Dr. rer. nat. degree (with distinction) in 1977.

That same year the DFG-Schwerpunkt Program (priority programme) "Digitale Signalverarbeitung" was established; it consisted of a group of some 45 communication engineers, geophysicists, seismologists and medical doctors which met ca. twice a year at various universities in order to discuss the research work achieved by their students. For the senior author, as a member of that group, the ideal student at his Lehrstuhl A für Mathematik was naturally Splettstößer. One of the many key problems dealt with by this unique priority programme was prediction theory, brought up particularly by the late H. W. Schüßler (Erlangen), Alfred Fettweis (Bochum) and H.D. Lüke. Wolfgang Splettstößer decided to tackle prediction theory and already in December of 1981 he presented his Habilitation thesis to the RWTH Faculty. In view of his fundamental research work in signal analysis as a whole he was nominated Professor (apl.) at the Lehrstuhl A für Mathematik in 1987. His work was basic for a number of the ca. 140 papers, doctoral and diploma theses written by members of the chair in the area up until 1994. As to the status of sampling theory in the year 1975 the reader may consult [21, pp. 29-30].

His interest in applied mathematics, in particular in signal processing theory, led Wolfgang Splettstößer to carry on his career in industry, namely in the field of microelectronic development, his first position in 1986 being a development engineer at Siemens Semiconductors, which became Infineon Technologies in 1999. He was promoted to Vice President of the Design-Center Düsseldorf in 1998, and finally took the role of the Senior Director of Human Resources at the Munich site in 2005. His deep concern for a dialogue-oriented management style motivated his continuous effort in the branch of "idea management", of which he became the global director of Infineon Technologies in 2007.

Furthermore, Wolfgang Splettstößer was director and member of the management board of the VDE (Association for Electrical, Electronic \& Information Technologies) 
Rhein Ruhr, from 2003 onwards. Some of his core interests, namely contacts between industry and universities, and a strong support of young researchers and engineers, for example as a selection-committee member for the "technics prize" awarded by the VDE Rhein-Ruhr, kept him connected with university during his whole career.

He passed away on March 18, 2013, and is survived by his spouse, Dr. Brigitte Splettstößer (née Koch), four daughters and a son.

Acknowledgments The authors would like to thank Professor Janine Splettstößer, Wolfgang's oldest daughter, for her contribution to the biography. Paulo Ferreira was partially supported by FCT under PTDC/EEA-TEL/108568/2008.

Open Access This article is distributed under the terms of the Creative Commons Attribution License which permits any use, distribution, and reproduction in any medium, provided the original author(s) and the source are credited.

\section{References}

1. Aldroubi, A., Gröchenig, K.: Nonuniform sampling and reconstruction in shift-invariant spaces. SIAM Rev. 43(4), 585-620 (2001). doi:10.1137/S0036144501386986 (electronic)

2. Apostol, T.M.: Modular Functions and Dirichlet Series in Number Theory, 2nd edn. Springer, New York (1990). doi:10.1007/978-1-4612-0999-7

3. Aronszajn, N.: Theory of reproducing kernels. Trans. Am. Math. Soc. 68, 337-404 (1950)

4. Beaty, M.G., Dodson, M.M.: Abstract harmonic analysis and the sampling theorem. In: Higgins, J.R., Stens, R.L. (eds.) Sampling Theory in Fourier and Signal Analysis, vol 2: Advanced Topics, chap. 10, pp. 233-265. Oxford University Press, Oxford (1999)

5. Beaty, M.G., Dodson, M.M.: The Whittaker-Kotel'nikov-Shannon theorem, spectral translates and Plancherel's formula. J. Fourier Anal. Appl. 10(2), 179-199 (2004). doi:10.1007/s00041-004-8010-6

6. Beaty, M.G., Dodson, M.M., Eveson, S.P.: A converse to Kluvánek's theorem. J. Fourier Anal. Appl. 13(2), 187-196 (2007). doi:10.1007/s00041-006-6025-x

7. Beaty, M.G., Dodson, M.M., Eveson, S.P., Higgins, J.R.: On the approximate form of Kluvánek's theorem. J. Approx. Theory 160(1-2), 281-303 (2009). doi:10.1016/j.jat.2009.02.013

8. Bellman, R.: A Brief Introduction to Theta Functions. Holt, Rinehart and Winston, New York (1961)

9. Boas Jr, R.P.: Summation formulas and band-limited signals. Tôhoku Math. J. 2(24), 121-125 (1972). doi: $10.2748 / \mathrm{tmj} / 1178241524$

10. Bochner, S.: Vorlesungen über Fouriersche Integrale. Chelsea Publishing Co., New York (1948)

11. Brown Jr, J.L.: On the error in reconstructing a non-bandlimited function by means of the bandpass sampling theorem. J. Math. Anal. Appl. 18(1), 75-84 (1967). doi:10.1016/0022-247X(67)90183-7

12. Brown Jr, J.L.: Erratum to: "On the error in reconstructing a nonbandlimited function by means of the bandpass sampling theorem". J. Math. Anal. Appl. 21, 699 (1968). doi:10.1016/ 0022-247X(68)90271-0

13. Brüdern, J.: Einführung in die analytische Zahlentheorie. Springer, Berlin (1995)

14. Butzer, P.L., Schmeisser, G., Stens, R.L.: The classical and approximate sampling theorems and their equivalence for entire functions of exponential type. J. Approx. Theory 179, 94-111 (2014). doi:10. 1016/j.jat.2013.11.010

15. Butzer, P.L., Ferreira, P.J.S.G., Higgins, J.R., Schmeisser, G., Stens, R.L.: The sampling theorem, Poisson's summation formula, general Parseval formula, reproducing kernel formula and the PaleyWiener theorem for bandlimited signals - their interconnections. Appl. Anal. 90(3-4), 431-461 (2011). doi:10.1080/00036811003627567

16. Butzer, P.L., Ferreira, P.J.S.G., Schmeisser, G., Stens, R.L.: The summation formulae of EulerMaclaurin, Abel-Plana, Poisson, and their interconnections with the approximate sampling formula of signal analysis. Results Math. 59(3-4), 359-400 (2011). doi:10.1007/s00025-010-0083-8

17. Butzer, P.L., Gessinger, A.: A decomposition theorem for Parseval's equation; Connections with uniform and nonuniform sampling. In: Bilinskis, I., Cain, G., Marvasti, F. (eds.) SampTA'95, pp. 100-107. Institute of Electronics and Computer Science, Riga (1995) 
18. Butzer, P.L., Gessinger, A.: The approximate sampling theorem, Poisson's sum formula, a decomposition theorem for Parseval's equation and their interconnections. Ann. Numer. Math. 4(1-4), 143-160 (1997)

19. Butzer, P.L., Jansche, S.: A direct approach to the Mellin transform. J. Fourier Anal. Appl. 3(4), 325-376 (1997). doi:10.1007/BF02649101

20. Butzer, P.L., Nessel, R.J.: Fourier Analysis and Approximation. Academic Press, New York; Birkhäuser Verlag, Basel (1971)

21. Butzer, P.L., Schmeisser, G., Stens, R.L.: An introduction to sampling analysis. In: Marvasti, F. (ed.) Nonuniform Sampling: Theory and Practice, pp. 17-121. Kluwer/Plenum, New York (2001). doi:10. 1007/978-1-4615-1229-5

22. Butzer, P.L., Splettstößer, W.: Approximation und Interpolation durch verallgemeinerte Abtastsummen. Forschungsberichte des Landes Nordrhein-Westfalen Nr. 2708. Westdeutscher, Opladen (1977)

23. Butzer, P.L., Stens, R.L.: The Euler-MacLaurin summation formula, the sampling theorem, and approximate integration over the real axis. Linear Algebra Appl. 52(53), 141-155 (1983). doi:10.1016/ 0024-3795(83)80011-1

24. Butzer, P.L., Stens, R.L.: The Poisson summation formula, Whittaker's cardinal series and approximate integration. In: Ditzian, Z., Meir, A., Riemenschneider, S.D., Sharma, A. (eds.) Second Edmonton Conference on Approximation Theory, pp. 19-36. American Mathematical Society, Providence (1983)

25. Deitmar, A.: A First Course in Harmonic Analysis, 2nd edn. Springer, New York (2005)

26. Dodson, M.M.: Approximating signals in the abstract. Appl. Anal. 90(3-4), 563-578 (2011). doi:10. 1080/00036811003627575

27. Doetsch, G.: Summatorische Eigenschaften der Besselschen Funktionen und andere Funktionalrelationen, die mit der linearen Transformationsformel der Thetafunktion äquivalent sind. Compositio Math. 1, 85-97 (1935)

28. Faridani, A.: A generalized sampling theorem for locally compact abelian groups. Math. Comp. 63(207), 307-327 (1994). doi:10.2307/2153577

29. Feldman, J., Greenleaf, F.P.: Existence of Borel transversals in groups. Pac. J. Math. 25, 455-461 (1968)

30. Ferrar, W.L.: Summation formulae and their relation to Dirichlet's series. Compositio Math. 1, 344-360 (1935). http://www.numdam.org/item?id=CM_1935_1_344_0

31. Flajolet, P., Gourdon, X., Dumas, P.: Mellin transforms and asymptotics: harmonic sums. Theoret. Comput. Sci. 144(1-2), 3-58 (1995). doi:10.1016/0304-3975(95)00002-E

32. García, A.G., Portal, A.: Sampling in reproducing kernel Banach spaces. Mediterr. J. Math. Published online: 28 Nov 2012. doi:10.1007/s00009-012-0234-0

33. Hamburger, H.: Über die Riemannsche Funktionalgleichung der $\xi$-Funktion. Math. Z. 10(3-4), 240-254 (1921). doi:10.1007/BF01211612

34. Hamburger, H.: Über die Riemannsche Funktionalgleichung der $\zeta$-Funktion. Math. Z. 11(3-4), 224-245 (1921). doi:10.1007/BF01203626

35. Hamburger, H.: Über die Riemannsche Funktionalgleichung der $\zeta$-Funktion. Math. Z. 13(1), 283-311 (1922). doi:10.1007/BF01485292

36. Hamburger, H.: Über einige Beziehungen, die mit der Funktionalgleichung der Riemannschen $\zeta$-Funktion äquivalent sind. Math. Ann. 85(1), 129-140 (1922). doi:10.1007/BF01449611

37. Han, D., Nashed, M.Z., Sun, Q.: Sampling expansions in reproducing kernel Hilbert and Banach spaces. Numer. Funct. Anal. Optim. 30(9-10), 971-987 (2009). doi:10.1080/01630560903408606

38. Hewitt, E., Ross, K.A.: Abstract Harmonic Analysis, vol. I: Structure of Topological Groups. Integration Theory, Group Representations. Springer, Berlin (1963)

39. Hewitt, E., Ross, K.A.: Abstract Harmonic Analysis, vol. II: Structure and Analysis for Compact Groups. Analysis on Locally Compact Abelian Groups. Springer, New York (1970)

40. Hewitt, E., Stromberg, K.: Real and Abstract Analysis. A Modern Treatment of the Theory of Functions of a Real Variable. Springer, Berlin (1965)

41. Higgins, J.R.: Sampling Theory in Fourier and Signal Analysis. Clarendon Press, Oxford (1996)

42. Higgins, J.R.: A sampling principle associated with Saitoh's fundamental theory of linear transformations. In: Saitoh, S., Hayashi, N., Yamamoto, M. (eds.) Analytic Extension Formulas and Their Applications (Collected papers 2nd Internat. Congress of the Internat. Society for Analysis, its Applications and Computation (ISAAC'99), Fukuoka, Japan, Aug. 16-21, 1999, and Research Meeting on Applications of Analytic Extensions, Kyoto, Japan, Jan. 11-13, 2000). Kluwer Academic Publishers, Dordrecht, pp. 73-86 (2001) 
43. Higgins, J.R.: Two basic formulae of Euler and their equivalence to Tschakalov's sampling theorem. Sampl. Theory Signal Image Process. 2(3), 259-270 (2003)

44. Higgins, J.R.: H. Hamburger's collection of five equivalent results, and some later developments, Technical Report, p. 31 (2007)

45. Higgins, J.R.: The Riemann zeta function and the sampling theorem. Sampl. Theory Signal Image Process. 8(1), 1-12 (2009)

46. Higgins, J.R.: Paley-Wiener spaces and their reproducing formulae. In: Ruzhansky, M., Wirth, J. (eds.) Progress in Analysis and Its Applications (Proceedings of 7th Internat. ISAAC Congress, London, Jul. 13-18, 2009, pp. 273-279. World Scientific Publisher, Hackensack (2010). doi:10.1142/ 9789814313179_0036

47. Hong, Y.M., Kim, J.M., Kwon, K.H.: Sampling theory in abstract reproducing kernel Hilbert space. Sampl. Theory Signal Image Process. 6(1), 109-121 (2007)

48. Ismail, M.E.H., Nashed, M.Z., Zayed, A.I., Ghaleb, A.F., eds. Mathematical Analysis, Wavelets, and Signal Processing, Proceedings of Internat. Conference on Mathematical Analysis and Signal Processing, Cairo, Jan. 3-9, American Mathematical Society, Providence, 1995, (1994). doi:10.1090/conm/ 190

49. Ivić, A.: The Riemann Zeta-Function. Wiley, New York (1985)

50. Kahane, J.P., Mandelbrojt, S.: Sur l'équation fonctionnelle de Riemann et la formule sommatoire de Poisson. Ann. Sci. École Norm. Sup. 3(75), 57-80 (1958)

51. Karatsuba, A.A., Voronin, S.M.: The Riemann Zeta-Function. Walter de Gruyter \& Co., Berlin (1992). doi:10.1515/9783110886146

52. Klusch, D.: The sampling theorem, Dirichlet series and Bessel functions. Math. Nachr. 154, 129-139 (1991). doi:10.1002/mana.19911540111

53. Klusch, D.: The sampling theorem, Dirichlet series and Hankel transforms. J. Comput. Appl. Math. 44(3), 261-273 (1992). doi:10.1016/0377-0427(92)90001-E

54. Kluvánek, I.: Sampling theorem in abstract harmonic analysis. Mat.-Fyz. Časopis Sloven. Akad. Vied 15, 43-48 (1965)

55. Knopp, M., Robins, S.: Easy proofs of Riemann's functional equation for $\zeta(s)$ and of Lipschitz summation. Proc. Am. Math. Soc. 129(7), 1915-1922 (2001). doi:10.1090/S0002-9939-01-06033-6

56. Krazer, A.: Lehrbuch der Thetafunktionen. Chelsea Publishing Co., New York (1970)

57. Mordell, L.J.: Poisson's summation formula and the Riemann zeta function. J. Lond. Math. Soc. S1-4(4), 285 (1929). doi:10.1112/jlms/s1-4.4.285

58. Murty, M.R.: Problems in Analytic Number Theory, 2nd edn. Springer, New York (2008)

59. Nashed, M.Z.: Inverse problems, moment problems, signal processing: un menage a trois. In: Siddiqi, A.H., Singh, R.C., Manchanda, P. (eds.) Mathematics in Science and Technology (Proceedings of Satellite Conference of the Internat. Congress of Mathematicians, New Delhi, India, Aug. 14-17, 2010). World Scientific Publishers, Hackensack, pp. 2-19 (2011)

60. Nashed, M.Z., Sun, Q.: Sampling and reconstruction of signals in a reproducing kernel subspace of $L^{p}\left(\mathbb{R}^{d}\right)$. J. Funct. Anal. 258(7), 2422-2452 (2010). doi:10.1016/j.jfa.2009.12.012

61. Nashed, M.Z., Sun, Q., Xian, J.: Convolution sampling and reconstruction of signals in a reproducing kernel subspace. Proc. Am. Math. Soc. 141(6), 1995-2007 (2013). doi:10.1090/ S0002-9939-2012-11644-2

62. Nashed, M.Z., Walter, G.G.: Reproducing kernel Hilbert spaces from sampling expansions. In: Ismail, M.E.H., Nashed, M.Z., Zayed, A.I., Ghaleb, A.F. (eds.) Mathematical Analysis, Wavelets, and Signal Processing, pp. 221-226 (1994). doi:10.1090/conm/190/02304

63. Newman, D.J.: Analytic Number Theory. Springer, New York (1998)

64. Nikol'skiĭ, S.M.: Approximation of Functions of Several Variables and Imbedding Theorems. Springer, New York (1975)

65. NuHAG (Numerical Harmonic Analysis Group). http://www.univie.ac.at/nuhag-php/home

66. Patterson, S.J.: An Introduction to the Theory of the Riemann Zeta-Function. Cambridge University Press, Cambridge (1988). doi:10.1017/CBO9780511623707

67. Pesenson, I.: Best approximations in a space of the representation of a Lie group (russian). Dokl. Akad. Nauk SSSR 302(5), 1055-1058 (1988). Trans. Soviet Math. Dokl. 38(2), 384-388 (1989)

68. Pesenson, I.: Sampling of Paley-Wiener functions on stratified groups. J. Fourier Anal. Appl. 4(3), 271-281 (1998). doi:10.1007/BF02476027

69. Pesenson, I.: A sampling theorem on homogeneous manifolds. Trans. Am. Math. Soc. 352(9), 4257-4269 (2000). doi:10.1090/S0002-9947-00-02592-7 
70. Pesenson, I.: Sampling of band-limited vectors. J. Fourier Anal. Appl. 7(1), 93-100 (2001). doi:10. 1007/s00041-001-0007-9

71. Pesenson, I.: Polynomial splines and eigenvalue approximations on quantum graphs. J. Approx. Theory 135(2), 203-220 (2005). doi:10.1016/j.jat.2005.04.007

72. Pesenson, I.: Sampling in Paley-Wiener spaces on combinatorial graphs. Trans. Am. Math. Soc. 360(10), 5603-5627 (2008). doi:10.1090/S0002-9947-08-04511-X

73. Reiter, H.: Classical Harmonic Analysis and Locally Compact Groups. Clarendon Press, Oxford (1968)

74. Riemann, B.: Über die Anzahl der Primzahlen unter einer gegebenen Größe. Monatsber. Königl. Preuss. Akad. Wiss. Berlin Nov. 1859, 671-680. Reprinted. In: Riemann, B., Narasimhan, R. (eds.) Gesammelte mathematische Werke, wissenschaftlicher Nachlass und Nachträge, (Based on the edition by Weber, H., Dedekind, R.). Springer, Berlin (1990)

75. Rooney, P.G.: Another proof of the functional equation for the Riemann zeta function. J. Math. Anal. Appl. 185(1), 223-228 (1994). doi:10.1006/jmaa.1994.1244

76. Rudin, W.: Fourier Analysis on Groups. Interscience Publishers, New York (1962)

77. Saitoh, S.: Integral Transforms. Reproducing Kernels and Their Applications. Longman, Harlow (1997)

78. Schuster, W.: Ein kurzer Beweis der Funktionalgleichung der Riemannschen Zetafunktion (a short proof of the functional equation of the riemann zeta function). Aequationes Math. 70(1-2), 191-194 (2005). doi:10.1007/s00010-004-2743-9

79. Siegel, C.: Bemerkung zu einem Satz von Hamburger über die Funktionalgleichung der Riemannschen Zetafunktion. Math. Ann. 86(3-4), 276-279 (1922). doi:10.1007/BF01457989

80. Stenger, F.: Numerical Methods Based on sinc and Analytic Functions. Springer, New York (1993)

81. Stenger, F.: Sinc convolution-a tool for circumventing some limitations of classical signal processing. In: Ismail, M.E.H., Nashed, M.Z., Zayed, A.I., Ghaleb, A.F. (eds.) Mathematical Analysis, Wavelets, and Signal Processing, pp. 227-240 (1994). doi:10.1090/conm/190/02305

82. Titchmarsh, E.C.: The Theory of the Riemann Zeta-Function, 2nd edn. Clarendon Press, New York (1986)

83. Weiss, P.: An estimate of the error arising from misapplication of the sampling theorem. Notices Am. Math. Soc. 10, 351 (1963). Abstract No. 601-54

84. Zayed, A.I.: Advances in Shannon's Sampling Theory. CRC Press, Boca Raton (1993)

85. Zygmund, A.: Trigonometric Series. vols. I, II, 2nd edn., reprinted with corrections and some additions (two volumes bound as one) edn. Cambridge University Press, London (1968) 